\title{
2. Die Politik der DEZA und das Beispiel einer NRO
}

Brigit Hagmann, Kathryn Imboden, Philippe Berberat, Daniel Fino and François Mercier

\section{(2) OpenEdition \\ 1 Journals}

Electronic version

URL: http://journals.openedition.org/sjep/831

DOI: $10.4000 /$ sjep.831

ISSN: 1663-9677

Publisher

Institut de hautes études internationales et du développement

\section{Printed version}

Date of publication: 1 avril 2001

Number of pages: $71-103$

ISSN: 1660-5926

\section{Electronic reference}

Brigit Hagmann, Kathryn Imboden, Philippe Berberat, Daniel Fino und François Mercier, « 2. Die Politik der DEZA und das Beispiel einer NRO », Schweizerisches Jahrbuch für Entwicklungspolitik [Online], 20 I 2001, Online erschienen am: 03 September 2012, abgerufen am 08 September 2020. URL : http:// journals.openedition.org/sjep/831; DOI : https://doi.org/10.4000/sjep.831 


\section{DIE POLITIK DER DEZA UND DAS BEISPIEL EINER NRO}

\subsection{PRIVATSEKTORFÖRDERUNG DER DIREKTION FÜR ENTWICKLUNG UND ZUSAMMENARBEIT}

Brigit Hagmann* und Kathryn IMBODEN*

\section{EINFÜHRUNG}

Arbeit, Einkommen und sichere Vermögensbildung sind wichtige Bedingungen für eine wirksame Armutsbekämpfung. Motor für wirtschaftliches Wachstum und die Schaffung von Arbeitsplätzen ist der private Sektor, während dem Staat die Aufgabe zukommt, für gute Rahmenbedingungen und eine gute Wirtschaftspolitik zugunsten eines armutsorientierten wirtschaftlichen Wachstums zu sorgen. Die DEZA setzt sich dafür ein, dass wirtschaftliches Wachstum den ärmsten Bevölkerungsgruppen auf dem Lande und in der Stadt zugute kommt. Starke private und öffentliche Institutionen und Organisationen sind die Voraussetzung für das Schaffen und Erhalten von Arbeitsplätzen. Landwirtschaftliche Produktion und Vermarktung, Unternehmensförderung, Finanzdienstleistungen und berufliche Bildung sind Instrumente zur wirksamen Einkommens- und Beschäftigungsförderung. Davon erwartet die DEZA langfristig einen Beitrag zu grösserer sozialer Gerechtigkeit. Die Programme der DEZA in den genannten Arbeitsfeldern haben zum Ziel, Kleinbetrieben, Haushalten und Kleinbauern/Kleinbäuerinnen den Zugang zu Dienstleistungen (Wissen und Fertigkeiten, Technologie, Finanzdienstleistungen, Marketing etc.) zu ermöglichen. Dies geschieht über starke oder entwicklungsfähige lokale Partner und Institutionen. Die DEZA unterstützt diese Partner in ihrer Entwicklung und lernt auch aus den Erfahrungen dieser Partner.

Nachfolgend wird aufgezeigt, wie die DEZA durch Unternehmensförderung in städtischen und ländlichen Regionen die Entwicklung des Privatsektors fördert, welches die Aufgabe des Staates dafür ist, und welche Instrumente sie dafür einsetzt. Nach einem Überblick über entwicklungspolitische Grundsätze und einem kurzen Abriss über Arbeitslosigkeit und Unterbeschäftigung, wird die Frage nach der Rolle des Staates und des Privatsektors gestellt. Wie weit Privatsektorförderung durch Unternehmensförderung zur Verminderung der Armut beiträgt, wird im nächsten Abschnitt diskutiert. Im darauffolgenden Teil A wird näher auf Unternehmensförderung durch Dienstleistungen zur Unternehmensentwicklung eingegangen, im Teil B steht Unternehmensförderung durch Finanzdienstleistungen im Vordergrund.

* Brigit Hagmann: Chefin der Sektion Arbeit und Einkommen, DEZA.

* Kathryn Imboden: Spezialistin des Finanzsektors, DEZA 
Aus dem «Bericht über die Politik der Entwicklungszusammenarbeit der Schweiz $1976-1985 »^{1}$ geht hervor, dass in diesen Jahren 33 von 39 Projekten der DEZA mit einem finanziellen Volumen von über 1 Mio. SFr. durch staatliche oder parastaatliche Partner durchgeführt wurden. Seit 1985 werden neben staatlichen Institutionen, Akteure der Zivilgesellschaft und private Institutionen und Unternehmungen zunehmend zu wichtigen Partnern der DEZA. Im Leitbild der DEZA² steht u.a.: «Zur Erfüllung unseres Auftrages unterstützen wir Personen, nichtstaatliche Organisationen und öffentliche Institutionen, welche lokal, auf Landesebene oder global wirken.» Die Stärkung der Zivilgesellschaft und die Förderung des Privatsektors wurden für die DEZA und andere Geberorganisationen zunehmend zu einem Ziel der Zusammenarbeit. Im Leitbild steht dazu: «Wir unterstützen benachteiligte Menschen, damit sie besseren Zugang zu Produktionsmitteln gewinnen und ihre Lebensbedingungen aus eigener Kraft verbessern können». In der Strategie 2010 der DEZA ${ }^{3}$, welche Steuerungshilfen für die Umsetzung des Leitbildes bietet, werden als Grundsätze festgehalten: Die DEZA strebt in ihrer Tätigkeit eine nachhaltige Entwicklung an. Die Programme der DEZA sind auf grösstmögliche Wirkung ausgerichtet. Sie unterstützen die Eigeninitiative der Partner in der Armutsminderung, im Abbau struktureller Konfliktursachen, in der Linderung von Not. Im Umgang mit immer schnelleren Veränderungen im globalen Umfeld orientiert sich die DEZA an globalen Herausforderungen. Die DEZA fokussiert ihre Aktivitäten auf fünf thematische Schwerpunkte: Gute Regierungsführung; Erhöhung der sozialen Gerechtigkeit; nachhaltige Nutzung der natürlichen Ressourcen; Einkommensförderung und Beschäftigung. Innerhalb des Schwerpunktes «Einkommensförderung und Beschäftigung» gehört die Privatsektorförderung durch Unternehmensentwicklung in ländlichen und städtischen Gebieten $\mathrm{zu}$ einer wichtigen Massnahme der DEZA gegen Arbeitslosigkeit und Unterbeschäftigung.

Übergeordnete Ziele für die Unternehmensentwicklung sind ${ }^{4}$ :

- langfristig wirksame Wachstumsimpulse geben, die den Bedürfnissen der Bevölkerung nach einem vermehrten und besseren Angebot an Gütern und Dienstleistungen entsprechen,

ป die Voraussetzungen schaffen, um stabile Arbeitsplätze und leistungsbezogene Einkommen zu erhalten und zu fördern,

a eine ausgeglichenere Verteilung des Sozialproduktes in der Bevölkerung und in den Regionen anstreben.

\section{$\square$ Arbeitslosigkeit und Unterbeschäftigung}

Unterbeschäftigung und Erwerbslosigkeit sind eines der Hauptprobleme für Entwicklungsländer und Industrieländer. Arbeit ist ein entscheidendes Element für die wirtschaftliche und soziale Entwicklung. Man rechnet, dass eine von drei

1. DEZA, Bericht über die Politik der Entwicklungszusammenarbeit der Schweiz 1976-1985, Bern 1987 (d,f,e).

2. DEZA, Leitbild der DEZA, Bern 1999 (d, f, i, e, sp).

3. DEZA, 2010 Strategie der DEZA, Bern, 2000 (d, f, i e, sp).

4. Aus: DEZA, Sektorpolitik Handwerks- und Industrieförderung, Bern 1992 (d, f, e, sp). 
Milliarden der aktiven Bevölkerung unterbeschäftigt oder arbeitslos ist. Zudem sind in vielen Ländern, vor allem in den DEZA-Schwerpunktländern, viele Beschäftigte ständig bedroht, ihren Arbeitsplatz zu verlieren; beinahe die Hälfte der aktiven Bevölkerung muss mit weniger als 2 US Dollar Verdienst pro Tag auskommen. Verschiedene UNO-Konferenzen haben die Regierungen zur Armutsbekämpfung aufgerufen. Die Teilnehmerländer haben sich darauf geeinigt, bis zum Jahre 2015 die Armut auf die Hälfte zu reduzieren. Wichtig war dieses Thema auch am Sozialgipfel in Genf im Juni 2000 (Kopenhagen + 5). Der Schaffung von Arbeitsplätzen und Einkommensquellen kommt eine zentrale Bedeutung zu, wobei dem Privatsektor die Rolle zukommt, die Arbeit zu schaffen, während der Staat dafür optimale Rahmenbedingungen bereitstellen sollte. Die Globalisierung führt zu sehr raschen Veränderungen der Wirtschaft und demzufolge $\mathrm{zu}$ einem sehr schnellen Anpassungsbedarf bei den Wirtschaftspartnern. Dies stellt enorme Herausforderungen an die Flexibilität der Arbeitenden sowie an deren Fähigkeiten und Möglichkeiten zur Aus- und Weiterbildung. Im ländlichen Raum kommt dazu, dass schwierige Umweltbedingungen, niedrige Ausbildung und schlecht angepasste Dienstleistungen die Anpassungsfähigkeit der Familienbetriebe noch weiter verringert. Die einseitigen Besitzverhältnisse verunmöglichen es grossen Teilen der Bevölkerung, die minimalsten Bedürfnisse zu decken und selbstversorgend zu leben. Obwohl die Landwirtschaft als arbeitsintensiver Sektor gilt, sind auch hier Grenzen gesetzt. Fehlende Arbeitsplätze in ländlichen Gebieten veranlassen die Bevölkerung zur Migration, womit sich die Probleme in den Städten und Agglomerationen weiter verschärfen. Die modernen, kapitalintensiven Industrie- und Dienstleistungssektoren schaffen trotz Wachstum zu wenig neue Arbeitsplätze. Im Zuge des Strukturwandels führen sie nicht selten zur Vernichtung traditioneller Arbeitsmöglichkeiten. Arbeitsplätze im öffentlichen Sektor nehmen stetig ab, neue werden v.a. im Privatsektor geschaffen. So sichern im Alltag Arbeitsplätze im informellen Sektor, in Handwerks-, Gewerbe- und kleinen Industriebetrieben das Überleben breiter Bevölkerungsschichten. ${ }^{5}$

\section{$\square$ Rolle von Staat und Privatsektor}

In allen Programmen ist die Klärung der Rolle des öffentlichen und des privaten Sektors von grösster Wichtigkeit. Starke private und öffentliche Institutionen und Organisationen sind die Voraussetzung für das Schaffen und Erhalten von Arbeitsplätzen. Der Motor für wirtschaftliches Wachstum und die Schaffung von Arbeitsplätzen ist der private Sektor, während dem Staat die Aufgabe zukommt für gute Rahmenbedingungen, eine gute Wirtschaftspolitik zugunsten eines armutsorientierten wirtschaftlichen Wachstums zu sorgen. Die Erfahrungen der DEZA zeigen, dass staatliche Institutionen wenig effizient sind in der Förderung von Privatunternehmen. Die DEZA arbeitet deshalb zunehmend mit privaten Institutionen und Dienstleistungszentren zusammen. Die DEZA betei-

5. Im Vorfeld des Sozialgipfels im Juni 2000 in Genf hat der Fachdienst «Industrie, Berufsbildung, Urbanisierung, (seit Oktober 2000 Teil der Sektion Arbeit und Einkommen) eine Tagung zum Thema «Arbeit gegen Armut» organisiert. Für diese Tagung wurde vom Fachdienst eine Broschüre mit demselben Titel herausgeben, in welcher veranschaulicht wird, welche Massnahmen die DEZA unterstützt zur Förderung von Einkommen und Beschäftigung. Ebenso wurde die internationale Situation in Bezug auf Arbeit und Einkommen analysiert und kurz dargestellt. DEZA, Arbeit gegen Armut, Bern 2000 (d, f, i, e, sp). 
ligt sich aber nicht direkt am Kapital von Privatfirmen, sondern setzt ihre Mittel über Förderinstitutionen ein. Dabei achtet die DEZA darauf, dass ihre öffentlichen Mittel nicht privates Risikokapital substituieren.

\section{$\square$ Armutsorientierung}

Die Förderung der Schaffung von Arbeitsplätzen und Einkommen ist eine notwendige, wenn auch nicht hinreichende Massnahme zur Bekämpfung der Armut. Armen Bevölkerungsgruppen soll einerseits der Zugang zu bestehenden Arbeitsplätzen und existenzsicherndem Einkommen ermöglicht werden (z.B. durch Berufsbildung), andererseits soll ein armutsorientiertes wirtschaftliches Wachstum gefördert werden, das neue Arbeitsplätze und Einkommen schafft. Die Geberländer sind sich heute einig, dass nicht jede Art von wirtschaftlichem Wachstum automatisch positive Wirkungen auf die Armutsverminderung hat («trickle down-Effekt»). Umgekehrt darf aber auch nicht geschlossen werden, dass die Schaffung von Arbeit und Einkommen und dadurch eine Verbesserung der sozialen Situation ohne wirtschaftliches Wachstum möglich ist. Zwischen landwirtschaftlicher Produktion und Vermarktung, Unternehmensförderung durch Finanz- und Nichtfinanzdienstleistungen und Berufsbildung können wichtige Synergien entstehen. Wege aus der Armut können beispielsweise mit der Gründung oder dem Beitritt zu einer Spargruppe beginnen, die später den Zugang zu Kredit öffnet und damit den Weg für Investitionen ebnet, so dass daraus Arbeit und Einkommen entstehen. Solche Prozesse werden in der Regel erleichtert, wenn in der Region ein arbeitsintensives, vor allem auch landwirtschaftliches Wachstum stattfindet, das neue Gelegenheiten für Arbeit und Einkommen bietet und damit auch das Gewerbe stimuliert. Der Motor einer solchen Entwicklung ist die private Wirtschaft, die auf gute Rahmenbedingungen angewiesen ist. Die wirtschaftliche Entwicklung kann mit gezielten Dienstleistungen wie Ausbildung, Management, Vermarktung, Technologien, Beratung, Finanzierung gefördert werden, die auf marktorientierter Basis angeboten werden. Ein wichtiger Aspekt zur Armutsbekämpfung ist dabei, die Transaktionskosten dieser Dienstleistungen tief zu halten und auf die Bedürfnisse der kleinen Betriebe, der Kleinbauern und all jener zuzuschneiden, die noch nie in den Genuss einer Ausbildung oder Förderung gekommen sind. Instrumente, die den Zugang zu Dienstleistungen auch ärmster Bevölkerungsschichten ermöglichen, müssen prioritär entwickelt werden.

Fragen nach den Wirkungsmechanismen, welche von der Unternehmensförderung zur Armutsreduktion führen, beschäftigen derzeit viele DEZA-Projekte. Der bisher klarste und erfolgreichste Ansatz ist eine Intervention auf Mesoebene durch die Förderung von Business Development Services (BDS). Allerdings stösst dieser Ansatz in sehr armen Gebieten auf Grenzen - eine Herausforderung, die wahrgenommen werden muss.

Der rasche Wandel der Gesellschaft bedingt auch eine Flexibilisierung der Ausbildung, da sich die Anforderungen ständig wandeln. Dazu gehören die Unterteilung der Ausbildungsinhalte in baukastenartig verknüpfte Module. Dies erleichtert den Zugang der Menschen zu Aus- und Weiterbildung. Auch kann bedarfsgerechter auf die Lebens- und Arbeitserfordernisse der Menschen eingegangen werden. Weiter erfordert dies ein dynamisches Verhalten der Institutionen, seien sie nun staatlich oder privat, um bedürfnis- und nachfragegerechte Ausbildungsangebote bereitzustellen. 
Die Unternehmensförderung gehört zu einem wichtigen Mittel im Kampf gegen die Armut, kann sie doch beitragen Arbeitsplätze zu erhalten und zu schaffen, wodurch Einkommen generiert wird, welches eine soziale Besserstellung ermöglicht. Aber Unternehmensförderung kann nur Eigeninitiativen unterstützen und muss erhebliche Eigenleistungen fordern. Dies gilt gegenüber dem Unternehmer/der Unternehmerin, gegenüber lokalen Förderinstitutionen und Dienstleistungszentren. Die DEZA will vermeiden, dieses Eigenleistungspotential lokaler Partner durch Geberkonkurrenzierung zu untergraben.

Das Potential für Unternehmensförderung ist besonders hoch, wenn Verflechtungen mit der Landwirtschaft und mit grösseren (exportorientierten) Industrien bestehen. Ländliche Unternehmensförderung erfordert gewisse Grundvoraussetzungen. Dazu gehören: eine relativ hohe Monetarisierung der Region, eine minimale Bevölkerungsdichte und die Verfügbarkeit zentraler Basisinfrastrukturen (Wasser, Elektrizität, Transport, Kommunikation).

Es sind aber auf verschiedenen Ebenen eine Reihe von Engpässen abzubauen, um vorhandene Potentiale besser ausschöpfen zu können.

Die Engpässe unternehmerischen Handelns lassen sich auf folgende Kernprobleme reduzieren : 6

- Rahmenbedingungen sind entwicklungsfeindlich und einflussnehmende Interessenverbände/Förderinstitutionen fehlen, sind ineffizient oder vertreten partikuläre Interessen einzelner Mitglieder.

- Beschaffungs- und Absatzmärkte sind fragmentiert, wenig transparent oder nicht zugänglich und es fehlt die Kaufkraft der Kunden.

- Es fehlt an fachlich qualifizierten Personen, die Unternehmen aufbauen und erfolgreich führen können. Der Mangel an erfahrenen und praktisch ausgebildeten Fachleuten senkt Produktivität und Qualität.

- Anlagen und Ausrüstungen sind nicht vorhanden, veraltet oder den lokalen Bedingungen nicht angepasst; der Zugang zu Wissen, Technologie und Finanzen ist erschwert oder versperrt.

\section{A UNTERNEHMENSFÖRDERUNG DURCH DIENSTLEISTUNGEN ZUR UNTERNEHMENSENTWICKLUNG (BUSINESS DEVELOPMENT SERVICES)}

\section{$\square$ Strategische Orientierung und Partner}

Projekte der Institutionenförderung (Aufbau, effiziente Führung und Vernetzung intermediärer Organisationen) und Projekte zur Entwicklung des Marktes für Dienstleistungen haben zum Ziel, den Klein- und Mittelunternehmen die nötigen Dienstleistungen (Business development services - BDS) zu marktgerechten Preisen verfügbar und zugänglich zu machen, und zwar Dienstleistungen, die sie brauchen, um ihr Unternehmen zu erhalten und weiterzuentwickeln. Partner für diese Interventionen (auf der Mesoebene) sind v.a. private intermediäre Organisationen (Business development centers), die in irgendeiner Form Kleinunternehmen aller Sektoren fördern.

Die DEZA interveniert meist nicht direkt auf der Ebene des Einzelbetriebs mit eigenen Programmen und eigenem Personal, weil es zu kostspielig und zeitrau-

6. DEZA, Sektorpolitik Handwerks- und Industrieförderung, Bern 1992 (d, f, e, sp). 
bend wäre, ein spezifisches Branchenwissen selber aufzubauen und weiterzuentwickeln. Sie beteiligt sich an solchen Programmen nur über kompetente Partner (Spezialorganisationen, Privatwirtschaft, gemeinnützige Privatorganisationen), denen sie die operationelle Verantwortung überträgt. Sie sucht diese Partner lokal oder international.

Interventionen auf der Makroebene zielen darauf ab, das Umfeld für unternehmerische Aktivitäten zu verbessern. Zu diesem Umfeld gehören die politischen, wirtschaftspolitischen, rechtlichen und institutionellen Rahmenbedingungen, also Faktoren, die den Handlungsspielraum Betroffener mitbestimmen, ohne dass sie selbst direkt darauf Einfluss nehmen können. Die DEZA interveniert auf dieser Ebene in der Regel in internationaler Absprache, um sicherzustellen, dass beabsichtigte Massnahmen breit abgestützt und wirkungsvoll sind.

\section{$\square$ Geografische Ausrichtung}

Programme zur Unternehmensförderung werden von der DEZA in allen Kontinenten unterstützt. Grundsätzlich kann in allen Ländern mit demselben Ansatz (BDS-Ansatz) gearbeitet werden, doch müssen die Instrumente von Land zu Land den jeweiligen wirtschaftlichen, politischen und sozialen Bedingungen angepasst werden. Im Jahr 2000 wurden in mehreren Regionen (Lateinamerika, Osteuropa) neue Projekte zur Vermarktung von landwirtschaftlichen Produkten eingerichtet und zahlreiche bestehende Projekte stärker auf den Markt ausgerichtet. Die Tatsache, dass neue Ansätze zugleich in mehreren Regionen angewendet werden, bietet die Gelegenheit, den Austausch und die Erfahrungskapitalisierung zwischen und innerhalb der Regionen zu fördern. Jährlich werden von der DEZA für Unternehmensförderungsprojekte ca. 25 Mio SFR zur Verfügung gestellt.

\section{$\square$ Marktorientierung statt Subventionen}

Die Unternehmensförderungsstrategie der DEZA gründet auf der Ansicht, dass Arbeitsplätze nicht durch Regierungserlasse und in einem von Entwicklungsprogrammen künstlich geschaffenen Klima entstehen und nachhaltig bestehen können, sondern nur durch unternehmerische Menschen und das Wertschöpfungspotential konkurrenzfähiger Unternehmen. Nur ein Unternehmen, das im Markt besteht, kann Beschäftigung sicherstellen. Die Unternehmensförderung darf deshalb das Beschäftigungsproblem nicht allein oder prioritär behandeln, ohne die Existenzfähigkeit des Unternehmers/der Unternehmerin respektive Unternehmens im Auge zu behalten.

Die Konsequenz, welche die DEZA daraus ableitet ist, dass Unternehmensförderung von der Marktorientierung, speziell von der Analyse der Nachfrage und Potentiale ausgehen muss. Erst anhand einer Marktanalyse können für ein konkretes Projekt Ziele und Strategie sowie angepasste personelle, finanzielle und technische Mittel bestimmt werden.

Das Überleben von Unternehmen ist vom Markt abhängig, und deshalb dürfen Unternehmen nicht durch Zuschüsse künstlich aufgebaut und am Leben erhalten werden. Die DEZA achtet darauf, dass von ihr unterstützte Aktionen keine Vergünstigungen an Unternehmen beinhalten, welche sich direkt auf die Produktkosten auswirken. Werden mit DEZA-Mitteln Leistungen an einzelne Unternehmen erbracht, so ist sicherzustellen, dass sie in angemessener Form abgegolten 
werden oder dafür dienen, eine spezifische Zielgruppe (z.B. Frauen) zu fördern. Die DEZA will damit die Begünstigung Einzelner verhindern.

Zuschüsse können vor allem dann angezeigt sein, wenn externe Erträge anfallen, d.h. wenn der volkswirtschaftliche Nutzen grösser ist als die betriebswirtschaftlichen Kosten der Massnahme (mögliche Beispiele sind Bildung und Beratung) oder zur Sicherstellung des Zugangs zu Dienstleistungen von Mikrounternehmen oder ärmeren Bevölkerungsschichten. Werden von der DEZA Zuschüsse gewährt, müssen deren Ziel, Umfang, Abwicklung und Dauer klar festgelegt werden. Es besteht die Gefahr, langfristige Abhängigkeiten von externen Finanzierungsquellen zu schaffen. Förderinstitutionen sind vor allem auf ihre professionelle Kompetenz und weniger auf ihre programmatischen Erklärungen zu prüfen. $\mathrm{Zu}$ viele Privatorganisationen sehen sich noch in der karitativ-sozialen Rolle anstatt als Anbieter kompetenter Dienstleistungen, die den Bedürfnissen von Unternehmen und Zivilgesellschaft entsprechen.

Die Forderung nach Professionalität und der Grundsatz der Funktionentrennung sprechen dafür, dass die Instrumente Ausbildung/Beratung einerseits und Kredit/Garantie andererseits zwar koordiniert eingesetzt, aber nicht durch ein und dieselbe Institution betreut werden.

Institutionen, deren professionelle Kompetenz erwiesen ist, werden vermehrt Programm- statt punktuelle Projektbeiträge gewährt, welche die fachliche Weiterentwicklung der Organisation fördern. Das bedeutet auch, dass mehr Ziel-Ergebnis-Vergleiche statt Durchführungskontrollen stattfinden.

\section{$\square$ Unternehmerinnenförderung}

Um Diskriminierungen zu verhindern, sind frauenspezifische Aspekte in jedem Unternehmensförderungsprojekt einzubeziehen. Dabei sind gewisse Differenzierungen nach Zielgruppen angezeigt. Im Mikrounternehmensbereich ist die Frauenquote hoch, und Dienstleistungszentren sind in der Regel entsprechend sensibilisiert für mögliche Diskriminierungen. Bei Klein- und Mittelunternehmensförderung müssen frauenspezifische Projektkomponenten sowohl auf der Mikro- wie Mesoebene sicherstellen, dass Unternehmerinnen als Zielgruppe wahrgenommen werden und ihnen gleichberechtigt Zugang zu Dienstleistungen ermöglicht wird. (Beispiele: spezielle Kreditfenster, Produkte und Lehrmaterial spezifischen Bedürfnissen anpassen, Berücksichtigung zeitlicher Verfügbarkeit). Ebenso muss sichergestellt werden, dass Informations- und Sensibilisierungsprogramme für die spezifische Situation von Mann und Frau im Unternehmen und im Privatbereich in die Projektaktivitäten einbezogen werden.

\section{$\square$ Angepasste Technologie}

Die DEZA fördert die Verwendung angepasster Technologien, d.h. eine in der konkreten Situation unter sozio-kulturellen und wirtschaftlichen Gesichtspunkten optimale Technologie. Die Frage, ob kapital- oder arbeitsintensive Verfahren zu fördern sind, ist nur unter Berücksichtigung der Marktlage möglich. Weil das Risiko schliesslich vom Unternehmer getragen werden muss, soll Unterstützung durch Beratung dem Unternehmer erlauben, verschiedene Alternativen und deren Risiken besser beurteilen zu können. 
Die DEZA achtet auf die Respektierung lokaler sozialpolitischer Grundsätze (z.B. Sozialversicherung, Abbau der Frauendiskriminierung, Mindestlöhne, Verbot ausbeuterischer Kinderarbeit, Arbeitssicherheit). Handwerk und Industrie sind in vielen Entwicklungsländern - gerade im Kleinunternehmensbereich für Mensch und Umwelt in hohem Masse belastend. In den von ihr unterstützten Unternehmensförderungsprogrammen achtet die DEZA auf die Einhaltung nationaler und internationaler Umweltvorschriften, fördert entsprechende technische Verbesserungen in Produktion und Entsorgung und unterstützt Massnahmen, die das Bewusstsein für Umweltfragen heben.

\section{$\square$ Zusammenarbeit auf internationaler Ebene}

Die DEZA beteiligt sich aktiv in den internationalen Fachgremien für Unternehmensförderung mit dem Ziel, den internationalen Erfahrungsaustausch aus Praxis und Forschung für die Verbesserung ihrer Aktivitäten zu nutzen, und um die Koordination unter Gebern und zwischen Gebern und Entwicklungsländern zu fördern. Das Geberkomitee für Unternehmensförderung 7 (es besteht seit 1979) hat nach mehrjähriger Zusammenarbeit im Oktober 2000 Richtlinien für Geberinterventionen im Bereich der Unternehmensförderung herausgegeben ${ }^{8}$. Diese Richtlinien stützen sich auf langjährige Erfahrung und «best practices» verschiedener Geber. Die DEZA war aktiv an der Entwicklung dieser Richtlinien beteiligt. Die Richtlinien halten fest, dass Unternehmensförderung nur erfolgreich sein kann, wenn sie von Anfang an marktorientiert gemacht und die Entwicklung eines Marktes für Dienstleistungen zum Ziel hat, die den Bedürfnissen der Unternehmen entsprechen. Subventionen sollen nur beschränkt und zeitlich limitiert gewährt werden und v.a. dazu dienen, finanzschwachen Unternehmerinnen und Unternehmern den Zugang zu Dienstleistungen zu ermöglichen.

\section{$\square$ Instrumente}

Die Aus-und Weiterbildung im technischen wie im unternehmerischen Bereich spielt in der Unternehmensförderung eine wichtige Rolle. Mit steigender Betriebsgrösse kommen der kaufmännischen Bildung und der Organisationsentwicklung wachsende Bedeutung zu. Beste technische Voraussetzungen und eine gesunde Marktlage sind nutzlos, wenn das Management versagt. Aus- und Weiterbildung werden von öffentlichen und privaten Ausbildungsinstitutionen oder von privaten Zentren zur Unternehmensentwicklung angeboten. Berufsbildungszentren werden teilweise langfristig von der DEZA mitsubventioniert und können deshalb finanziell günstigere Angebote machen als Privatanbieter. Es ist jedoch wichtig, dass auch die Nutzniesser (Betriebe, Auszubildende) finanzielle Leistungen erbringen, da sie von dieser Aus- oder Weiterbildung rasch und direkt profitieren. Private Ausbildungsanbieter (Business development center) hingegen müssen marktkonforme Preise für ihre Ausbildungsangebote verlan-

7. DEZA/Weltbank, Committee of Donor Agencies for Small Enterprise Development: What is it?, What does it do?, Bern 2000 (e).

8. Weltbank/Committee of Donor Agencies for Small Enterprise Development, Business development services for SME's: Preliminary guidelines for Donor-Funded Interventions, Washington 1998 (e). Definitive Richtlinien wurden vom Komitee im Oktober 2000 verabschiedet und anfangs 2001 von der Weltbank publiziert. 
gen, um finanzielle Nachhaltigkeit zu erreichen oder zu behalten. Um sicherzustellen, dass auch finanzschwächere Unternehmerinnen Zugang zu diesen Angeboten haben, werden heute häufig von Regierungen oder Geberorganisationen Ausbildungsgutscheine (Vouchers) zur Verfügung gestellt, die einen Teil der Ausbildungskosten decken. (Eine Studie zur Aufarbeitung der Erfahrungen mit Ausbildungsgutscheinen in Asien und Lateinamerika ist in Erarbeitung9).

Individuelle Betriebsberatung ist teuer und wird deshalb auf der Stufe der Mikro- und Kleinunternehmen nur bei relativ dichtem Klientennetz angewendet. Berater sind lokale Fachpersonen und nicht internationale Experten. Dadurch werden sprachliche/kulturelle Hindernisse abgebaut und Kosten gesenkt. Kompetente Beratung setzt fachliche, pädagogische und kommunikative Fähigkeiten voraus. Internationale Experten werden beinahe ausschliesslich für die Aus- und Weiterbildung von lokalen Beratern eingesetzt. Wie für Ausbildung, soll für Beratung auch eine Kostenbeteiligung der Klienten von Projektbeginn an verlangt werden. Durch Kostenbeteiligung werden nicht nur die Programmkosten gesenkt, sondern sie ist auch der einfachste und aussagekräftigste Erfolgsindikator eines Beratungsprogrammes. Zudem sind die Berater motivierter und kundenorientierter, wenn sie ihren Klienten Rechnung stellen müssen und die Klienten kritischer im Einfordern von qualitativ hochstehender Beratung. Business development center, die marktorientiert arbeiten, sind selten in der Lage, für Kleinunternehmen kostendeckende Beratung zu erschwinglichen Preisen anzubieten.

Die Stärkung von Interessenverbänden und cluster ist ein wichtiges Instrument der Unternehmensförderung. Voraussetzung für den nachhaltigen Aufbau von Verbänden sind dauerhafte, klar erkennbare Vorteile für die organisierten Personen. Wo diese nicht bestehen, hat der Verband keine Zukunft. Bewährt hat sich die gruppendynamische Methode, um gemeinsame Interessen zu identifizieren und die Solidarität untereinander zu fördern. Wichtige fachliche Funktionen von Interessenverbänden sind: Schulung/Beratung, Normenwesen, Erfassung und Auswertung von Branchenkennziffern, Information über Fachmessen etc.

Die DEZA fördert in den von ihr unterstützten Bereichen den Aufbau von Netzwerken. Netzwerke organisieren den Informationsaustausch, die Harmonisierung von Standards und Verfahren und die Arbeitsteilung zwischen verschiedenen Institutionen. Die DEZA setzt sich vor allem auf der Mesoebene für die Stärkung des Wissens und Erfahrungsaustausches zwischen Entwicklungsländern ein, um lokale Kapazitäten zu stärken.

Der Politikdialog mit Regierungen kann Gegenstand der Unternehmensförderung sein, wenn Rahmenbedingungen die Fördermassnahmen auf der Mikro- und Mesoebene behindern. Im konkreten Fall ist festzulegen, ob die Erfüllung bestimmter Rahmenbedingungen Kriterium der Aufnahme/ Fortsetzung der Tätigkeit in einem Land/einem Projekt sein soll (Konditionalität), oder ob die Verbesserung der Rahmenbedingungen Bestandteil des Projektes sein soll.

Der Entscheid über inhaltliche Aspekte und Vorgehensweise auf der Makroebene erfolgt in der Regel in internationaler Absprache.

9. Die Voucherstudie wird von Swisscontact, Zürich, durchgeführt und von der DEZA kofinanziert. Sie kann ab Frühling 2001 bei der DEZA oder bei Swisscontact bezogen werden. 


\section{$\square$ Impaktmessung}

Um die Wirksamkeit der Projekte zur Unternehmensförderung zu kennen, arbeitet die DEZA zusammen mit anderen nationalen und internationalen Geberorganisationen an einem Instrument, das Impakt, Wirkungsbereich und Nachhaltigkeit der Interventionen messen soll. Nicht nur Geber sollen ein Messinstrument erhalten, sondern auch Intermediärorganisationen und Unternehmungen sollen anhand einfacher Indikatoren ihre Fortschritte und Leistungen messen können. Es ist schwierig, kostengünstig zuverlässige Daten zu erheben, um quantifizierbare Aussagen darüber zu machen, wie sich Unternehmensförderungsprojekte auf die Schaffung von Arbeitsplätzen und Einkommen und dadurch auf die Verminderung der Armut auswirken. Eine internationale Arbeitsgruppe, bestehend aus Mitgliedern des Committee of Donor Agencies for Small Enterprise Development, unter der Leitung der Internationalen Arbeitsorganisationen (ILO) ist daran, Indikatoren und Instrumente zu entwickeln und zu testen, die in diesen Projekten angewendet werden könnten. Erste Resultate dieser Arbeitsgruppen sollten bis Ende 2001 vorliegen.

\section{B UNTERNEHMENSFÖRDERUNG DURCH FINANZDIENSTLEISTUNGEN}

\section{Strategie mit zwei Schwerpunkten}

Die Entwicklungszusammenarbeit strebt die nachhaltige Entwicklung lokaler Finanzinstitutionen an, die in einer guten Ausgangslage sind, um den Bedürfnissen einer finanzschwachen Kundschaft nachzukommen. Ihr Ansatz ist einerseits, den Zugang zu den Finanzdienstleistungen für die Kunden, die bis dahin vom formellen Finanzsystem ausgeschlossen waren, zu erweitern, und andererseits die Entwicklung von langfristig existenzfähigen Finanzintermediären zu unterstützen.

\section{Armutsbekämpfung durch Ermöglichung}

des Zugangs zu Finanzdienstleistungen

Die Programme im Finanzdienstleistungsbereich haben das gleiche Zielpublikum, das die DEZA auch in anderen Bereichen anvisiert, nämlich die bedürftigen Bevölkerungsgruppen in den Schwerpunktländern. Die Programme zur Unterstützung von Finanzdienstleistungen sind grundsätzlich auf sehr arme Bevölkerungsschichten ausgerichtet. Doch dienen die Finanzdienstleistungen für kleine und mittlere Unternehmen auch dazu, den Wohlstand armer Bevölkerungsgruppen durch die Schaffung von Arbeitsplätzen zu fördern. Die innovativen Finanzinstitutionen und -organisationen sind immer mehr imstande, einem Kundenkreis, der bislang von den Dienstleistungen der formellen Banken ausgeschlossen war, angepasste Dienstleistungen anzubieten. Und es wird jetzt anerkannt, dass der Zugang zu zuverlässigen Finanzdienstleistungen wichtiger ist als der Preis solcher Dienstleistungen.

\section{$\square$ Besonderer Fokus auf die Frauen}

Die Förderung von Finanzdienstleistungen für Frauen hat-dopppelte positive Auswirkungen. Die Steigerung des Einkommens der Frauen führt zu wirtschaftlichen und sozialen Verbesserungen. Die Frauen sind gut gestellet, um von inno- 
vativen Finanzdienstleistungen zu profitieren und erfolgreiche Mikro- und Kleinunternehmen zu gründen. Zudem verbessern sie mit der Verwendung ihres Einkommens zum Wohl ihrer Familien (Ernährung, Gesundheits- und Erziehungsdienstleistungen) ihre soziale Situation. Es ist bekannt, dass die Frauen als Kundinnen für die gute Einhaltung ihrer Verpflichtungen bekannt sind und deshalb als zuverlässig und kreditwürdig eingeschätzt werden.

\section{$\square$ Förderung der Entwicklung existenzfähiger Finanzinstitutionen}

Es wird anerkannt, dass die Erhöhung des Angebots an tragfähigen Finanzdienstleistungen durch die Stärkung von Institutionen erfolgt, die solche Dienstleistungen anbieten können, und nicht durch die Unterstützung punktueller Aktionen im Rahmen der Projekte. Nur eine Berücksichtigung der Leistungsfähigkeit und langfristig der Lebensfähigkeit lokaler Institutionen wird dauerhafte Lösungen ermöglichen. Die Stärkung der Institutionen und die Ausarbeitung von Betriebsplänen, welche die operationelle und danach die finanzielle Unabhängigkeit dieser Institutionen anstreben, sind die einzigen Mittel, um den Zielbevölkerungsgruppen auf lange Sicht helfen zu können.

Die DEZA hat sich den Richtlinien angeschlossen, die von der Gesamtheit der Geber im Dokument «Micro and Small Enterprise Finance: Guiding Principles for Selecting and Supporting Intermediaries" (Finanzierung von Klein- und Mikrounternehmen: Richtlinien zur Auswahl und Unterstützung von Intermediären ${ }^{10}$ ) anerkannt werden. Diese Richtlinien haben zum Ziel, gemeinsame Normen für die Geber auszuarbeiten, welche Klein- und Mikrounternehmen den Zugang zu Finanzdienstleistungen erleichtern wollen. Der strategische Rahmen für die Unterstützung strebt gerade diese beiden oben erwähnten komplementären Ziele an.

\section{$\square$ Programme der DEZA}

Die DEZA bringt jährlich über 10 Mio. SFR für Programme und Projekte im Bereich der Finanzdienstleistungen (u.a. Mikrofinanzierungen) auf. Die Beiträge der DEZA sind im allgemeinen nicht rückzahlbar; sie stellt in gewissen Fällen Darlehen und Garantien bereit und beteiligt sich ausnahmsweise am Gesellschaftskapital. Die DEZA fördert auch Ausbildung, Workshops für Erfahrungsaustausch, Studien und Initiativen zur Förderung des Politikdialogs.

\section{$\square$ Förderinstrumente}

Zur optimalen Berücksichtigung der verschiedenen Ausgangssituationen und der diversen wirtschaftlichen und sozialen Verhältnisse vor Ort setzt die DEZA entsprechend angepasste Förderinstrumente punktuell oder in Kombination ein. Diese sollen unter Einhaltung der festgelegten Richtlinien zu folgenden Zielen beitragen:

๑ die Partnerorganisationen organisatorisch, institutionell und finanziell fördern;

10. Dieses Dokument wurde vom Geberkomitee für Unternehmensförderung und von der Arbeitsgruppe der Geber über die Finanzsektorentwicklung im März 1996 ausgearbeitet. 
口 innovative Technologien zur Herabsetzung der Kosten auf der Nachfragewie auch auf der Angebotsseite identifizieren, erproben, wo nötig anpassen und schliesslich verbreiten;

口 eine Minimierung der Risiken ermöglichen.

\section{$\square$ Institutionelle Entwicklung}

Die Unterstützung zu Fragen der Organisationsentwicklung einer Partnerinstitution hat eine wichtige Funktion und kann im Übrigen im Verlauf der Zusammenarbeit verschiedene Formen annehmen. Indem die Förderung sich auf die beim Partner selbst vorhandenen Kräfte abstützt, kann sie im Laufe der Zeit sehr selektiv und punktuell werden. Unter den hierfür vorgesehenen Instrumenten sind folgende zu nennen: Organisationsentwicklung, Beratung, strategische und technische Betreuung, berufliche Aus- und Fortbildung von Direktion, Kaderleuten und Personal. Ein wichtiger Faktor ist die Unterstützung bei der Einführung der Verbesserung von Kontroll- und Managementinstrumenten (z.B. Managementinformationssysteme, MIS) und bei der Informatisierung (Hardund Softwareprogramme).

\section{$\square$ Beteiligung durch Kapitaleinlage}

Unter entsprechender Beachtung und Abwägung der Risiken kann die DEZA sich ausnahmsweise am Eigenkapital von Finanzinstitutionen beteiligen, um die nötige Kapitalgrundlage und die Erreichung der entwicklungspolitisch wichtigen Ziele bei der Gründung einer Institution und ihrer späteren Entwicklung zu gewährleisten. Die Beteiligungen durch Kapitaleinlagen können auch eingesetzt werden, um zusätzliche lokale Ressourcen zu mobilisieren.

\section{$\square$ Darlehen}

Darlehen können sich als sehr nützlich erweisen, um einem mittelfristigen Mangel an Finanzmitteln abzuhelfen, weshalb die DEZA solche Darlehen gewährt. Sie garantiert, dass zuerst einmal alle Finanzierungsmöglichkeiten auf lokaler Ebene geprüft werden. Die konkreten Bedingungen sollen so ausgehandelt werden, dass weder Marktverzerrungen noch Vorzugssituationen auftreten. Die DEZA trifft rechtzeitig die erforderlichen Massnahmen für Kapitalrückflüsse und deren Verwendung.

\section{$\square$ Garantiefonds}

Unter bestimmten Bedingungen können Kreditgarantien die Gewährung von Krediten durch Finanzinstitutionen erleichtern. Jedoch sind sie nur selten wirksame Instrumente, um den Kreditzugang für das Zielpublikum dauerhaft zu garantieren. Die DEZA kann die Kosten für die Erprobung und die Schaffung institioneller Kreditgarantieinstrumente dort übernehmen, wo ihr Einsatz geeignet ist, Engpässe beim Kreditzugang zu überwinden.

\section{$\square$ Kredit- und Rotationsfonds}

Wo solche Fonds bereits bestehen, muss dafür gesorgt werden, dass sie professionell, das heisst durch im Kreditbereich kompetente Mitarbeitende und gemäss den Kriterien des Finanzsystems verwaltet werden, und dass sie gemäss einem strengen Zeitplan zu angepassten Institutionen führen. Wo trotz allem 
mangels Alternativen ein neuer Fonds geschaffen wird, muss dies im Hinblick auf eine baldige Institutionalisierung und in Übereinstimmung mit der Politik des Finanzsektors erfolgen.

\section{$\square$ Kontakte und Partnerschaften}

Eine weitere Aufgabe zur Unterstützung des Finanzsystems kann darin liegen, Finanzinstitutionen mit bestehenden Selbsthilfe-, Kunden- oder Intermediärorganisationen (beispielsweise NRO) in Kontakt zu bringen, um die Transaktionskosten zu reduzieren und die Risiken zu decken (Gruppendruck). Die DEZA kann bei der Ermittlung von Partnern und bei der Aushandlung von Partnerschatsverträgen Hilfe leisten.

\section{Ausbildungsinstitutionen}

Die DEZA kann bankenexterne Ausbildungsorganisationen für die Mitarbeitenden der Finanzinstitutionen und/oder für die Kundinnen und Kunden unterstützen und stärken.

\section{$\square$ Meinungsaustausch, Zusammenarbeit und Studien}

Es ist notwendig, nationale, regionale und internationale Netzwerke, Seminare und Konferenzen sowie Meinungsaustausch und deren Konkretisierung zu unterstützen. Die (Ko)Finanzierung oder die Durchführung von Sektorstudien manchmal auch länderspezifisch können sich von grosser Bedeutung für die Entwicklung von Programmen und Sektoren erweisen.

\section{$\square$ Politikdialog und Unterstützung der Reformen}

Die DEZA pflegt, soweit möglich gemeinsam mit anderen Geldgebern, einen regelmässigen Politikdialog mit der Regierung des Partnerlandes und beteiligt sich auch an den Reformen des Finanzsektors.

\section{$\square$ Geografische Konzentration}

Die DEZA unterstützt eine Vielfalt von Institutionen in Afrika (vor allem Westafrika), Südasien, Lateinamerika, Osteuropa und in der früheren Sowjetunion (siehe Rahmentext). Ihre Unterstützung von Women's World Banking gestattet es, dieses globale Netzwerk von Mikrofinanzinstitutionen zu stärken, das den Zweck hat, den Bedürfnissen einkommensschwacher Unternehmerinnen nachzukommen.

Die DEZA ist auf der Ebene nationaler und internationaler Netzwerke tätig, insbesondere bei der «Beratungsgruppe der Hilfe für die Ärmsten», einem Konsortium aller bilateralen und multilateralen Geber im Mikrofinanzbereich.

\section{SCHLUSSFOLGERUNGEN}

Privatsektorförderung ist für die Umsetzung der Strategie 2010 der DEZA ein wichtiges Instrument und die Ansätze dafür, die in den letzten Jahren entwickelt wurden, werden auch in den kommenden Jahren angewendet und verfeinert. Höchste Priorität hat dabei die Überprüfung der Ansätze und Instrumente in Bezug auf deren Ausrichtung auf das Hauptziel der DEZA, nämlich die Verminderung der Armut. Der Zugang der DEZA-Zielgruppen (die ärmsten Bevölke- 
rungsgruppen) zu wichtigen Dienstleistungen und Produkten steht im Vordergrund der weiteren Instrumentenentwicklung. Bis heute können nur wenig quantitative Aussagen über die Wirkung der Unternehmensförderungsprogramme gemacht werden. Deshalb wird die DEZA in den nächsten Jahren versuchen, in enger Zusammenarbeit mit anderen Geberorganisationen und Nichtregierungsorganisationen Indikatoren und Messinstrumente zu entwickeln, die in allen Projekten angewendet werden können. Die Zusammenarbeit mit anderen Gebern und der ILO am Projekt «measurement of job creation » wird voraussichtlich im nächsten Jahr erste Grundlagen für das Vorgehen bei der Messung, ob Arbeitsplätze geschaffen werden, liefern. Eine grosse Herausforderung ist die Anpassung der Ansätze und Instrumente, die sich in Entwicklungsländern bewährt haben, an die Situation und Anforderungen der Länder in Osteuropa und Zentralasien. Zusammen mit der Sparte Zusammenarbeit mit Osteuropa und der GUS werden nächstes Jahr die in diesen Ländern gemachten Erfahrungen kapitalisiert und daraus nötige weitere Schritte für die Entwicklung von angepassten Strategien und Instrumenten zur Privatsektorförderung festgelegt.

\section{Beispiele $^{11}$}

Indien - Seidenproduktion

Wenn eine Kleinbauernfamilie eine Hektare Maulbeerblätter anpflanzt, werden für die Seidenproduktion insgesamt 13 Arbeitsplätze geschaffen, nämlich: Für den Anbau der Maulbeerbäume, die Aufzucht der Raupen, das Haspeln, Zwirnen, Färben und Weben. Indiens gesamte Maulbeer-Anbaufläche von 312'000 ha schafft somit Arbeit für vier Millionen Menschen. Zum Vergleich: eine Hektare Zuckerrohr schafft gerade 1,5 Arbeitsplätze.

Die DEZA unterstützt die Seidenproduktion in Indien seit über 20 Jahren. Dieser Sektor ist sehr arbeitsintensiv, und Seide hat einen grossen (Binnen-) Markt. Mit Unterstützung der DEZA wurde ein Ausbildungszentrum aufgebaut, das "International Centre for Training and Research in Tropical Sericulture (ICTRETS) ». Neben zahlreichen indischen Fachkräften bilden sich hier Spezialisten aus weiteren Ländern Asiens, Afrikas und Lateinamerikas aus. Indien ist mit 14'000 t Rohseide hinter China der weltweit zweitgrösste Seidenproduzent. Kein anderer landwirtschaftlicher Betriebszweig schafft so viele Arbeitsplätze.

Die Förderung der Seidenproduktion ist ein wichtiger Beitrag zur wirtschaftlichen Entwicklung im ländlich-bäuerlichen Raum. Sie schafft Arbeit für Kleinbauern und sichert ein regelmässiges Einkommen. Mehr als die Hälfte der Arbeit in der Seidenraupenzucht wird von Frauen geleistet. Das Einkommen verbessert ihre wirtschaftliche Situation, verschafft ihnen Ansehen und Respekt in Familie und Gesellschaft und erlaubt ihnen, über ihr eigenes Leben zu entscheiden.

Maulbeerpflanzen und Seidenraupen sind lebendige Organismen. Weder der Anbau noch die Aufzucht lassen sich vollständig industrialisieren. Deshalb wird die Seidenherstellung auch in Zukunft auf menschliche Arbeitskraft und kleingewerbliche Strukturen angewiesen sein. Diese beschäftigungsintensive Produktion ermöglicht Kleinbetrieben eine nachhaltige technische Entwicklung und unternehmerische Initiative und schafft somit wirtschaftliche Entwicklungsmöglichkeiten für mehrere Millionen Menschen in Indien.

Die DEZA unterstützt alle Stufen der Seidenverarbeitung, vom verbesserten Saatgut, resistenteren Raupensorten, besseren Verarbeitungsmethoden der Rohseide bis zu produktiveren Färbe- und Webmethoden. Eine wichtige Rolle spielen zudem soziale und ökologische Fragestellungen: Einbezug der Frauen, Abbau der Kinderarbeit, Verbesserungen beim Bleichen und Färben, Optimierung der Energienutzung.

11. Aus : DEZA, Arbeit gegen Armut, Bern 2000 (d, f, i, e, sp). 
Die Investitionen der DEZA beliefen sich zwischen 1980 und 1994 auf rund 5 Mio. Franken. Mit 4 Mio. Franken unterstützte die DEZA zudem die Verarbeitung der Seidenproduktion in Andhra Pradesh und Tamil Nadu. Das bisher grösste Engagement der DEZA war die Kofinanzierung des "National Sericulture Projects" der Weltbank von 1989 bis 1996. Ziel war die Verbesserung der Qualität und der Produktivität der Seidenzucht in fünf Gliedstaaten. Dies wurde erreicht durch bessere Ausbildung und durch die Stärkung der Stellung der Frauen im Produktionsprozess. Die Weltbank investierte rund 200 Mio. Franken, die Schweiz rund 30 Mio. Franken.

\section{Rumänien - Kleingewerbeförderung}

Ziel der Kleingewerbeförderung in Rumänien ist es, den marktwirtschaftlichen Reform- und Aufbauprozess zu unterstützen. Nutzniesser sind fähige und engagierte rumänische Fachkräfte, welche die Möglichkeit erhalten, selbständige Gewerbebetriebe aufzubauen. Der schweizerische Beitrag unterstützt das rumänische Kleingewerbe in der Vermittlung von Kleinkrediten, der Beschaffung von geeigneten Maschinen sowie in der fachlichen und betriebswirtschaftlichen Ausbildung. Projektpartner in Rumänien sind die gemeinnützigen Organisationen ROMCOM und der viele Kleinbetriebe umfassende Gewerbeverband Immanuel. Seit 1991 hat die Christliche Ostmission jährlich ca. 25 Betriebe ausgerüstet und deren Inhaber ausgebildet. Betriebe mit bis zu 12 Mitarbeitenden und Lehrlingen sind entstanden, die heute selbständig arbeiten. Insgesamt sind so über 1400 Arbeitsplätze geschaffen worden. Die DEZA unterstützt dieses Projekt seit 1993.

\section{Benin - Selbsthilfe der Frauen}

Im Juni 1995 waren in Cotonou, der Hauptstadt des afrikanischen Kleinstaats Benin, 12 Sparkassen mit insgesamt 1'436 Mitgliedern zusammengeschlossen. Die Einlagen betrugen 7,1 Mio. FCFA bzw. umgerechnet 17'750 Franken.

Ende 1998 waren es bereits 23 Sparkassen mit 4'343 Mitgliedern und 42,6 Mio. FCFA bzw. 106'500 Franken an Einlagen. Die Spar- und Leihkassen sind zwei bis dreimal pro Woche geöffnet und befinden sich in einer Schule, einem Gesundheitszentrum oder auch mal unter einem Baum.

Wie die meisten afrikanischen Städte, so wächst auch Cotonou stark. Quartier reiht sich an Quartier, ohne Strom oder Wasser. Kaum jemand hat eine feste Anstellung, meist wird der Kampf ums Überleben der Familie den Frauen überlassen. Dank der Verarbeitung von landwirtschaftlichen Produkten oder durch Näharbeiten, gelingt es den Frauen, das Nötigste für die Familie zusammenzubringen. Diese Unternehmungen erfordern meist ein bescheidenes Startkapital, welches kaum je aufgebracht werden kann, da andere Verpflichtungen - z.B. die Versorgung von kranken Kindern - den Vorrang haben. Dieser Zustand führte 1994 zur Einführung eines einfachen Spar- und Kreditsystems, das quartierweise und von den Frauen selbst organisiert wurde.

Die DEZA, die bereits ein Alphabetisierungsprogramm für Frauen unterstützte, beteiligte sich an diesem Pilotprojekt ("Association d'Entraide des Femmes»). Sie unterstützte die Frauen bei der Entwicklung ihrer Organisation. Strategische Entscheidungen trafen die Frauen selber.

Von Anfang an war klar, dass die DEZA die Kredite nicht subventionierte.

Kaderleute und Personal wurden in Weiterbildungskursen die nötigen Kenntnisse vermittelt, damit diese fähig waren, ihre Sparkasse nach betriebswirtschaftlichen Kriterien zu führen. Die Sparkassen erhielten den juristischen Status einer einfachen Gesellschaft. Dank diesen Mikrofinanzinstituten konnten Frauen, die bisher keinen Zugang zu Krediten hatten, ihre beruflichen Tätigkeiten ausbauen, ihre Lebenssituation verbessern und sich für ihre eigenen Interessen selber einsetzen.

\section{Bolivien - Finanzsektorreform}

Ein funktionierendes Bankenwesen auf dem Lande ist eine wichtige Voraussetzung für eine gedeihliche Wirtschaft, vor allem für Gewerbe und Landwirtschaft und damit für die Schaffung von Arbeitsplätzen. In Bolivien geht es darum, die bestehenden Finanzinstitutionen mehr auf die Bedürfnisse der Kundinnen und Kunden auszurichten. Nicht befriedigt wird beispielsweise die Nachfrage nach Finanzdienstleistungen der Kleinindustrie und der Landfrauen. Deshalb entschied die DEZA, die bestehenden Finanzinstitutionen (private Finanzfonds, im Kreditbereich tätige Nichtregierungsorganisationen und Spar- und Kre- 
ditgenossenschaften) in drei Bereichen zu unterstützen: Entwicklung neuer Mikro-Finanzprodukte (Ersparnisse, Mikro-Kredit, "Leasing " etc.), effizientere Kreditvermittlung (Reduktion der Transaktionskosten) und Ausbildung des Personals. Partner dieses Programms sind 75 Finanzfonds und 30 Nichtregierungsorganisationen.

Die Beschäftigungswirksamkeit einer solchen Intervention auf der gesamtwirtschaftlichen Ebene ist nicht direkt messbar. Doch sind verbesserte Rahmenbedingungen die beste Grundlage für ein wirtschaftliches Wachstum, vor allem im ländlichen Raum.

Albanien - Gründung von Spar- und Kreditvereinen

Über eine Kofinanzierung mit der Weltbank (IDA) finanziert die DEZA seit 1992 die Entwicklung von Mikrofinanzdienstleistungen in Albanien. In einer ersten Phase hat die DEZA ein Finanzdienstleistungsprogramm im Bezirk Puka im Norden Albaniens durch die Finanzierung einer Kreditlinie unterstützt, die den dörflichen Kreditfonds dieses Bezirks vom "Albanian Development Fund" angeboten wurde. Was den Agrarkredit betrifft, so wurde dieser Fonds durch den "Rural Finance Fund" ersetzt, eine quasi regierungseigene Stiftung, die dazu bestimmt ist, die Gründung von Spar- und Kreditvereinen, in Wirklichkeit die Privatisierung des dörflichen Kreditfonds-Netzes zu unterstützen, um ein langfristig existenzfähiges Netzwerk daraus zu machen. In der Übergangsphase zu einem selbständigen System von Spar- und Kreditvereinen finanziert die DEZA Entwicklungs- und Ausbildungsaktivitäten.

\section{Women's World Banking}

Die DEZA finanziert auch globale Initiativen, darunter Women's World Banking. Women's World Banking ist eine 1979 gegründete nicht profitorientierte Finanzinstitution mit dem Ziel, die wirtschaftliche Beteiligung von Frauen auf der Ebene der Mikro- und Kleinunternehmen zu fördern. Mit einem Netz von 46 angeschlossenen Mitgliedern (" affiliates") in 39 Ländern ist Women's World Banking das erste globale Netzwerk, das dazu bestimmt ist, den Kreditzugang für Frauen zu öffnen. Die Kundinnen sind Unternehmerinnen in Industrie, Handel und Landwirtschaft; die Mitglieder des Netzwerks sind lokale Finanzinstitutionen, welche Finanzdienstleistungen und Dienstleistungen zur Unterstützung der Unternehmer erbringen. WWB/New York, Dienstleistungszentrum für das Netz und Kommunikationsdrehscheibe, stellt institutionelle, technische und finanzielle Fördermassnahmen sowie eine Plattform für Erfahrungsaustausch und gemeinsames Lernen bereit. Ausserdem ist WWB dafür bekannt, Fortschritte im Bereich gesetzlicher und verordnungsrechtlicher Rahmenbedingungen zu initiieren, welche die Entwicklung des Mikrofinanzgewerbes ermöglichen.

\section{BASIX Indien - Alternatives ländliches Bankwesen}

BASIX ist eine Institution der neuen Generation zur Förderung ländlicher Einkommen, die 1996 in Hyderabad, Südindien, gegründet wurde. BASIX's Aufgabe ist, eine grosse Anzahl nachhaltiger Lebensunterhaltseinkommen - einschliesslich für die arme Landbevölkerung und die Frauen - durch die Bereitstellung von Finanzdienstleistungen und technischer Hilfe in einem integrierten Ansatz zu fördern. BASIX ist bestrebt, ihren Investoren eine konkurrenzfähige Rendite einzubringen, um dadurch Zugang zum Hauptanteil an Kapital und menschlichen Ressourcen auf kontinuierlicher Basis haben zu können. BASIX ist eine Gruppe von Gesellschaften, die Finanzdienstleistungen und technische Hilfe erbringen. Bhartiya Samruddhi Finance Limited (Samruddhi), welche bei der Reserve Bank of India als Nichtbanken-Finanzierungsgesellschaft mit beschränkter Haftung registriert ist, ist die Hauptbetriebseinheit, durch welche die Kredite vergeben werden. Indian Grameen Services (IGS) ist eine Nichtregierungsorganisation, die technische Hilfe und Unterstützungsdienstleistungen für Samruddhi-Darlehensnehmer sowie andere Agrarproduzenten und Institutionen bereitstellt. Beide werden durch die Holdinggesellschaft BASICS Ltd. zusammengehalten, über die die Gründungskapitalbeteiligungen an Samruddhi getätigt wurden.

BASIX hat mit einer Reihe innovativer Finanzprodukte durch unterschiedliche Kanäle experimentiert und dadurch den Zugang benachteiligter Gruppen zu Finanzdienstleistungen verbessert. Neben der direkten Darlehensvergabe an Kunden gewährt BASIX auch Darlehen durch Organisatoren im Bereich der Saatguterzeugung, Vertreter für die Vermarktung landwirtschaftlicher Produkte, Selbsthilfegruppen etc. BASIX's Mitarbeiter im Aussendienst und im Dienstleistungsbereich bringen den Leuten das Bankge- 
schäft an die Türschwelle. BASIX hat 10'000 aktive Kunden und besitzt einen Darlehensbestand von rund 5,4 Mio. SFR. Die Zusammenarbeit mit BASIX hat auch eine Auswirkung auf das gesamte Finanzsystem gehabt. BASIX spielt eine wichtige Rolle bei der Festlegung der Firmenpolitik betreffend die aktive Beteiligung an bedeutenden Foren wie Microfinance Task Force und Sa-Dhan, ein Dachverband von Mikrofinanzinstitutionen in Indien. Zudem ist BASIX unter den ersten indischen Finanzierungsgesellschaften des Nichtbankensektors, die im Mikrofinanzbereich tätig sind. BASICS hat auch andere NRO unterstützt, um die Bereitstellung von Finanz- und Nichtfinanzdienstleistungen zu modernisieren. Schliesslich hat die Reserve Bank of India beschlossen, BASICS als Gütezeichen eine ("local area») Banklizenz zu erteilen, und somit wird BASICS unter den ersten drei lokalen Banken in Indien sein.

BASICS wurde seit ihrer Gründung von der DEZA mit einem Darlehen und einem Zuschuss für technische Hilfe unterstützt.

\section{QUELLEN}

DEZA, Sektorpolitik Finanzwesen, Bern 1998 (d, f, e, sp).

DEZA, Sektorpolitik Handwerks- und Industrieförderung, Bern 1992 (d, f, e, sp).

DEZA/Weltbank, Committee of Donor Agencies for Small Enterprise Development: What is it?, What does it do ?, Bern 2000 (e).

DEZA, Arbeit gegen Armut, Bern 2000 (d, f, i, e, sp).

DEZA, Bericht über die Politik der Entwicklungszusammenarbeit der Schweiz 1976-1985, Bern 1987 (d, f, e).

DEZA, SCDs ' CIP/SED Program in the Nineties: Review and Challenges ahead, Issue Paper no. 4, Bern 1999 (e).

DEZA, Leitbild der DEZA, Bern 1999 (d, f, i, e, sp).

DEZA, 2010 Strategie der DEZA, Bern, 2000 (d, f, i e, sp).

Weltbank/Committee of Donor Agencies for Small Enterprise Development, Business development services for SME's: Preliminary guidelines for Donor-Funded Interventions, Washington 1998 (e).

Committee of Donor Agencies for Small Enterprise Development and Donor's Working Group on Financial Sector Development; «Micro and Small Enterprise Finance Guiding Principles for Selecting and Supporting Intermediaries», 1995 (e,f,sp). 


\title{
2.2. DIE ENTWICKLUNG DES INSTRUMENTS DER INTERNATIONALEN BANKGARANTIE IM DIENSTE DER ÄRMSTEN
}

\author{
Philippe Berberat, Daniel Fino, François Mercier*
}

\section{EINLEITUNG}

In fast allen Entwicklungsländern wird der grösste Teil der Wirtschaftstätigkeit im so genannten informellen oder nichtstrukturierten Sektor abgewickelt. Handwerker, Händler, Agrarproduzenten, Züchter usw., die allein oder in kleinen Gruppen arbeiten, sichern den Hauptanteil der Produktion, der Vermarktung und des Vertriebs der alltäglichen. Konsumgüter. Das wesentliche Merkmal und gleichzeitig auch der Vorteil des informellen Sektors ist, dass er sich aus Kleinbzw. Kleinststrukturen zusammensetzt: einfache Werkstätten, ein (pro Einheit) geringer Geschäftsumfang, ein im Durchschnitt eher bescheidenes Geschäftseinkommen und Umsatzvolumen, eine begrenzte Anzahl von Arbeitsplätzen. Aber der informelle Sektor wirft auch Probleme auf: neben der Tatsache, dass der Sektor sich praktisch jeglicher Besteuerung entzieht, werden die Einkommen hauptsächlich für den Verbrauch verwendet und nicht wiederinvestiert, was ein ziemlich rudimentäres Ausrüstungsniveau zur Folge hat. Aufgrund seiner informellen Organisationsform, seiner auf den lokalen Markt beschränkten Tätigkeit, seiner relativ niedrigen Produktivität usw. ist es für den informellen Sektor schwierig, seine Aktivitäten auszudehnen (um zumindest für einen nationalen Binnenmarkt zu produzieren, ohne zwangsläufig an die Ausfuhr zu denken), eine signifikante Wertsteigerung zu schaffen und sich der Konkurrenz massiver Einfuhren aus anderen Entwicklungsländern zu stellen.

Eines der grossen Hindernisse, das es dynamischen wirtschaftlichen Akteuren des informellen Sektors verwehrt, zu investieren, neue Aktivitäten zu entwickeln und $\mathrm{zu}$ innovieren, ist die Schwierigkeit, Zugang zu den erforderlichen Finanzmitteln, mit anderen Worten Zugang zum Kredit zu erhalten.

In den letzten Jahren hat das Thema der Mikrofinanzierung - das heisst die Frage der Mobilisierung, der Zugänglichkeit und der Verwendung finanzieller Ressourcen für die informelle Wirtschaft - grosse Aktualität erlangt. Es gibt viele Vorschläge und Erfahrungen, die diskutiert werden ${ }^{12}$, die wir aber in diesem Artikel nicht behandeln können.

Wir beschränken uns hier auf ein bestimmtes Instrument, das in der modernen Wirtschaft sehr gut bekannt ist, nämlich die Garantie ${ }^{13}$. In welchem Rahmen

* Philippe Berberat: Stiftung RAFAD, Sonderberater.

Daniel Fino: Stiftung RAFAD, Vorsitzender des Exekutivkomitees.

François Mercier: Stiftung RAFAD, Direktionsmitglied.

12. Die Überlegungen betreffen die Instrumente, die zu entwickeln sind, um die Finanzkraft der Ärmsten zu fördern, ferner die für die Mikrofinanzinstitutionen anzuwendenden Bedingungen, die Rolle der alternativen Banken, die Suche nach Versicherungs- und Leasingformen für den informellen Wirtschaftssektor, usw.

13. Die bekannteste Form ist der Hypothekarkredit, bei dem eine Immobilie der Bank, die dem Eigentümer Geld leiht, als Sicherheit dient. Ein anderes Beispiel, das der uns hier interessierenden Problematik näher steht, ist ein Garantiefonds, der in der Zwischenkriegszeit in der Schweiz von Handwerksverbänden mit Hilfe des Bundes gegründet wurde, um für Projekte, die den Banken unterbreitet werden, Bürgschaft zu leisten. Der Fonds besteht weiterhin. 
kann die Garantie in den Entwicklungsländern eine Rolle spielen ? Zur Behandlung dieser Frage müssen wir die Überlegungen auf die verschiedenen möglichen Situationen ausdehnen, mit denen die Kreditnehmer konfrontiert sind:

1. Es gibt keine Finanzinstitutionen, die Kleinunternehmern des informellen Sektors Finanzmittel leihen können.

2. Die modernen Banken vor Ort tätigen ihre Geschäfte mit den Akteuren des modernen Sektors und gewähren den Akteuren des informellen Sektors keinen Zugang.

3. Es gibt Spar- und Kreditsysteme (lokale Kassen, Spar- und Kreditgenossenschaften, Hilfskassen auf Gegenseitigkeit, usw.), die Kredite gewähren können.

4. Es gibt internationale Entwicklungshilfeorganisationen, die subventionierte Kredite (zu Vorzugsbedingungen) vergeben.

5. Es gibt traditionelle Organisationsformen (beispielsweise Leibrentengemeinschaften), die Spargelder mobilisieren und wieder verteilen.

6. Die Kreditnehmer wenden sich an Wucherer, mit all den damit verbundenen Problemen (übermässige Kosten).

Das Garantieinstrument kommt nur bei den Situationen 2 und 3 in Betracht. Unabhängig vom Bankensystem (Geschäftsbank, Genossenschaftsbank, alternative Bank, usw.) ist jede Institution, die Geld verleiht, bestrebt, sich durch Garantien (Hypotheken oder andere Bürgschaftsformen) abzusichern. Dies geschieht, um zu vermeiden, dass die Leihanstalt bei Nichtrückzahlung des Kredits Verluste erleidet. In den Entwicklungsländern, besonders bei den Akteuren der informellen Wirtschaft, bildet der Garantiemangel das Haupthindernis, um die Finanzierung zu finden. Wir sprechen hier von «bankfähigen» Projekten, die folglich allen geforderten Kriterien entsprechen. Es handelt sich auch um Projekte, die bei der sozio-ökonomischen Entwicklung des Landes eine wichtige Rolle spielen können, weil sie in technischer, wirtschaftlicher und sozialer Hinsicht interessant sind, indem sie für Innovationen aufgeschlossen sind und zur Schaffung von Einkommen und Beschäftigung beitragen.

Um den Finanzmittelzugang für Akteure zu erleichtern, die nicht in der Lage sind, die notwendigen Garantien zu leisten, kann die Einrichtung von Garantiefonds eine Lösung sein. Es gibt verschiedene Typen und Formen von Garantiefonds ${ }^{14}$, aber das Prinzip ist immer das gleiche: es gibt einen Fonds, der aus flüssigen Mitteln, Wertpapieren, Bürgschaftsbriefen von natürlichen oder juristischen Personen, usw. besteht. Dieser Fonds ist gesperrt und dient dazu, Kredite zu garantieren, die von einer Finanzinstitution vergeben werden. Der Einsatz einer Garantie verursacht Kosten, die zu den Kreditkosten hinzuzufügen sind.

Kann das Garantieinstrument dazu beitragen, das Problem des Kreditzugangs für Kleinproduzenten und Kleinhändler der informellen Wirtschaft in den Entwicklungsländern zu lösen? Um diese Frage zu beantworten, führen wir das Beispiel einer Schweizer Institution, der Stiftung RAFAD ${ }^{15}$ an, die seit fünfzehn

14. Fonds, die bei einer Bank in dem Land deponiert sind, wo die Darlehen vergeben werden; Fonds ausserhalb des Landes; Fonds, die von den Leihanstalten selbst verwaltet werden; von unabhängigen Organisationen verwaltete Fonds, usw. Die Garantiefonds, die in den Entwicklungsländern zum Einsatz kommen, werden in der Regel von der internationalen Entwicklungszusammenarbeit unterstützt, namentlich um die Betriebskosten zu decken.

15. RAFAD (Recherches et Applications de Financements Alternatifs au Développement), Stiftung schweizerischen Rechts, die 1985 von Charles A. Egger, Bernard Faure, Mahamian Kipiangah, Bernard Lecomte und Fernand Vincent gegründet wurde. 
Jahren mit diesem Instrument arbeitet. Der Ansatz wird durch zwei Fallstudien veranschaulicht. Wir schliessen unseren Artikel mit umfassenderen Überlegungen über die Garantie $a b$, da der Einsatz dieses Instruments für die Entwicklungsländer umstritten ist.

\section{URSPRUNG UND ENTWICKLUNG VON RAFAD}

Die Stiftung RAFAD wurde vor rund fünfzehn Jahren gegründet. Ihre Gründer verfolgten Ziele verschiedener Art, die aber alle innovativ und ambitiös waren. Unter den wichtigsten Zielen sind zu nennen:

- die Veränderung der Entwicklungshilfebeziehungen, um die Rolle des Schenkungsfaktors in der internationalen Entwicklungszusammenarbeit zu verringern, der die Selbsthilfe nicht fördert, und um dem Entwicklungshilfesystem eine indirektere und bescheidenere Rolle bei der Unterstützung der internen Dynamik in den Ländern einzuräumen;

- die Einführung von Finanzinstrumenten der modernen Wirtschaft für den informellen Wirtschaftssektor;

- die Mobilisierung der örtlich verfügbaren Finanzmittel (nationale Spargelder) zu Gunsten der lokalen Organisationen, indem man ihnen den Zugang zum Kredit eröffnet;

- die Stärkung der Kompetenzen dieser Organisationen zur Verwaltung von Fonds (durch Ausbildung und Begleitmassnahmen);

- die angewandte Forschung und die Reflexion über Entwicklungsfinanzierungsfragen (durch Studien, durch die Verbreitung der Experimente und durch Information).

RAFAD konnte mit der Vergabe von Garantien beginnen dank der Mittel, die eines der Gründungsmitglieder zur Verfügung gestellt hatte, und durch die Bereitstellung einer Rückbürgschaft ${ }^{16}$ durch den Bund. Die Stiftung steht seit ihrer Gründung in engem Kontakt mit einem bedeutenden Netzwerk von Partnern: nationale NRO, Verbände, Spar- und Kreditkassen, Basisgruppen, usw. Um nahe dem Tätigkeitsfeld vor Ort zu arbeiten und sich einer grösseren Objektivität bei der Bewertung der Dossiers zu vergewissern, verfügt RAFAD über lokale Berater, die mit Leistungsverträgen in den Ländern arbeiten, wo Garantien ausgegeben werden. Sie sichern die Verbindung mit dem Genfer Büro und unterhalten Beziehungen zu Partnern und Basisgruppen, um die erforderlichen Informationen zusammenzutragen, die Garantieantragsdossiers zu vervollständigen, die bereitgestellten Angaben zu prüfen und zu bewerten, mit der Bank zu verhandeln, die Betreuung sicherzustellen, usw.

Um RAFAD's Erfahrung Dauerhaftigkeit zu verleihen, indem man die Garantievergabestruktur zu einer von den Garantienehmern kontrollierten Gesellschaft weiterentwickelte, wurde 1996 eine Genossenschaft «Fonds International de Garantie (FIG)» gegründet. RAFAD befindet sich derzeit in der Phase, wo sie dem FIG ihre gesamte Garantietätigkeit überträgt, was auch den Vorteil haben

16. Dabei handelt es sich um ein Schreiben, in dem der Bund sich verpflichtet, für eventuelle Verluste aufzukommen, die sich infolge der Ausstellung von durch RAFAD gewährte Garantien ergeben könnten. Das Schreiben wird bei der Bank hinterlegt, welche die Ausstellungsaufträge von RAFAD ausführt. 
wird, die Forschungs- und Beratungstätigkeit besser vom Garantiesystem zu trennen. Der FIG, der von RAFAD verwaltet wird und an ihre Stelle treten wird, arbeitet in der gleichen Marktlücke, in der RAFAD bis heute tätig war. Der wesentliche Unterschied ist, dass nur die Mitglieder der Genossenschaft FIG eine Garantie erhalten können und dass der Geschäftsanteil der Mitglieder bei Inanspruchnahme ihrer Garantie herangezogen wird. Somit muss man, um eine Garantie zu erhalten, zwangsläufig Mitglied des FIG sein und Geschäftsanteile am FIG erworben haben.

Vor Gewährung einer Garantie besteht der wichtigste Teil der Arbeit darin, das Risiko einzuschätzen. Verschiedene Faktoren werden bei der Risikoabschätzung berücksichtigt, wie:

๖ das institutionelle Risiko (die «Qualität» des Partners, seine Verwaltungsund Betreuungskapazität, der Wille zur Transparenz, die Erfahrung im betreffenden Bereich, usw.);

$\checkmark$ das wirtschaftliche Risiko (die Durchführbarkeit des Projekts, seine Rentabilität, die Kreditrückzahlungsrate, usw.);

」 das finanzielle Risiko (Kapitalisierung, finanzielle Stabilität der NRO);

๖ das Länderrisiko (politische, finanzielle, sozio-ökonomische Rahmenbedingungen, lokale Gesetzgebung, usw.);

๖ das Bankrisiko (Zusammenarbeit mit der Lokalbank);

๖ $\quad$ sonstige Risiken (Transport, Korruption, usw.).

Zwischen 1985 und September 2000 liegt die globale Verlustrate von RAFAD/FIG im Durchschnitt bei 5,6\% pro Jahr der ausgestellten Garantien.

Ein zweiter wichtiger Aspekt, der vor der Garantiegewährung untersucht werden muss, ist die erwartete Hebelwirkung (Leverage-Effekt), das heisst der Betrag, der dank der Garantiegewährung schliesslich im Wirtschaftskreislauf in Umlauf gebracht wird. Durchschnittlich ist die Hebelwirkung bei RAFAD über 3, das heisst dass für 1 Franken Garantie 3 Franken Kredite vergeben wurden.

Ein dritter Faktor, der bei der Beurteilung des Dossiers berücksichtigt wird, ist die Auswirkung des Projekts auf die Armutsminderung. Wieviel Arbeitsplätze können durch den Kredit geschaffen werden, der dank der Garantie vergeben wird? Welche Einkommen und Spareinlagen werden hervorgebracht? Wie kann die Lokalgemeinschaft Nutzen daraus ziehen? Wir wissen, dass Tausende von Menschen Arbeit finden und ihr Einkommen verbessern konnten, doch ist die Auswirkung auf die Minderung der Armut schwer abzuschätzen. RAFAD verfügt über eine Reihe von Daten, die wir in den nachfolgenden Beispielen vorfinden werden, aber auch in Evaluationen, die (im August 1997) durchgeführt wurden.

Die Garantie ist natürlich nicht das einzige Instrument, um die Wirtschaftstätigkeiten an der Basis zu fördern. Aber in spezifischen Situationen kann sie ein angepasstes, ja sogar unumgängliches Instrument sein, um Akteuren der informellen Wirtschaft den Zugang zu lokal verfügbaren Finanzmitteln zu ermöglichen. Die nachstehend zusammengefassten Fallstudien geben einen kleinen Überblick über die Verschiedenartigkeit der Situationen, in denen die Garantie eine Rolle spielen kann. 


\section{Einige Angaben über RAFAD/FIG}

\begin{tabular}{|c|c|}
\hline Grösse des Garantiefonds: & 3'960'228 SFR (davon RAFAD: $3.506^{\prime} 158$ F, FIG : 454'070 F) \\
\hline Mitgliederzahl (FIG): & 23 Personen und Organisationen \\
\hline Ausgestellte Garantien: & 1'749'668 SFR (davon RAFAD: 1.575'248 F, FIG: 174'420 F) \\
\hline Anzahl Dossiers: & $\begin{array}{l}-18 \text { (davon RAFAD: } 15, \mathrm{FIG}: 3 \\
\text { - Dossiers in Bearbeitung: } 6\end{array}$ \\
\hline Vertretung vor Ort: & $\begin{array}{l}\text { Lokale Berater in } 10 \text { Ländern (davon } 7 \text { Länder mit laufenden } \\
\text { Projekten). }\end{array}$ \\
\hline $\begin{array}{l}\text { Geographische Verteilung } \\
\text { der Garantien: }\end{array}$ & $\begin{array}{l}\text { Mittelamerika: } 58 \% \text {, } \\
\text { Südamerika: } 4 \% \text {, } \\
\text { Afrika: } 38 \%\end{array}$ \\
\hline $\begin{array}{l}\text { Durchschnittsbetrag } \\
\text { einer Garantie: }\end{array}$ & 97'000 SFR (54'000 USD) \\
\hline Profil der Partnerorganisationen: & $\begin{array}{l}\text { - Direkte Kunden: } \\
\text { - Organisationen zur Unterstützung von Kleinst-, Klein- und } \\
\text { Mittelbetrieben (43\% des Bestands) } \\
\text { - Spar- und Kreditorganisationen und Mikrofinanzinstitutionen (14\%) } \\
\text { - Bauernorganisationen (43\%) } \\
\text { Indirekte Kunden: Kleinst-, Klein- und Mittelbetriebe im } \\
\text { Handwerks-, Agrar- und Handelssektor, } \\
\text { die auf den internationalen und lokalen Märkten tätig sind }\end{array}$ \\
\hline
\end{tabular}

\section{ZWEI FALLSTUDIEN MIT DER GARANTIE DES RAFAD-SYSTEMS}

- Beispiel: RAFAD's Partnerschaft mit dem Kreditund Sparkassennetz Nyesigiso in Mali

In Mali, einem der ärmsten Länder der Welt, ist seit 1989 ein beeindruckendes Netz an lokalen Kredit- und Sparkassen entstanden. Nyesigiso wurde von einer kanadischen NRO initiiert und gefördert und konnte ab 1997 auch auf RAFAD's Hilfe in Form einer Bankgarantie zählen. Zehn Jahre nach seiner Gründung umfasst das Netz nicht weniger als 44 Kassen und rund 50'000 Mitglieder. 1999 konnte Nyesigiso auf RAFAD's Unterstützung verzichten und hat die Garantie zurückgegeben.

\section{Eine paradoxale Wirtschaftslage}

Die makroökonomische Lage in Mali Mitte der 90er Jahre war durch schwere Behinderungen gekennzeichnet, wobei noch die Tatsache hinzukam, dass der informelle Wirtschaftssektor $80 \%$ bis $90 \%$ der Volkswirtschaft Malis ausmachte. Unter diesen Umständen war die Erlangung von Krediten bei den malischen Finanzinstitutionen für den informellen Sektor äusserst schwierig, während die Geschäftsbanken ihrerseits über grosse Überliquiditäten verfügten. 


\section{Ein auf Vertrauen basierendes Netz}

Nyesigiso, das ausschliesslich mit dem informellen Sektor arbeitet, setzt sich gegenwärtig aus unabhängigen lokalen Kassen zusammen, die Spargelder einsammeln und Kredite vergeben. Das Sparsystem weist die Besonderheit auf, dass die Einlagen nicht verzinst werden, da das Hauptmotiv für den Beitritt zur Kasse das Sicherheitsgefühl ist. Was den Kredit betrifft, so wird er nur an Kassenmitglieder und unter strengen Auflagen sowie mit Garantien (dingliche Sicherheit oder Bürgschaft) vergeben. Das auf Vertrauen beruhende System verzeichnet eine sehr hohe Rückzahlungsrate, da auf die schlechten Zahler ein starker sozialer Druck ausgeübt wird. Die Darlehensbeträge sind bei Einzeldarlehen auf 500'000 CFAF (1'250 SFR) und bei Gruppendarlehen auf 700'000 CFAF (1'750 SFR) begrenzt; die Dauer beträgt 12 Monate oder darunter, der Zinssatz liegt etwas über dem der Geschäftsbanken (12\% pro Jahr).

Man kann noch hervorheben, dass Nyesigiso eine gute Finanzlage aufweist. Das Netz hat nämlich 1996 und 1997 bedeutende Gewinne erzielt, es verfügt über genügend Eigenkapital und hat praktisch keine Kapitalkosten, da die Spareinlagen nicht durch Zinsen vergütet werden.

\section{Eine RAFAD-Garantie zur Stärkung von Nyesigiso's Kreditvergabekapazität}

Bei Nyesigiso war das Argument, sich an RAFAD zu wenden, die Tatsache, dass einer der Partner des Netzes (la Caisse des Entrepreneurs) damals einen beträchtlichen Zuwachs an Kreditanträgen verzeichnete, dabei aber vor zwei Hindernissen stand. Zum einen verfügte er nicht über genügend Kapital, um seine eigenen Kredite zu gewähren; zum anderen untersagte eine der Regeln von Nyesigiso, mehr als einen gewissen Prozentsatz der in anderen Kassen gesammelten Spargelder zu seinen Gunsten zu verwenden.

Somit ermöglichte es die Lösung einer Bankgarantie, Zugang zu einem Darlehen ausserhalb des Netzes zu erlangen und dadurch der starken Kreditnachfrage zu entsprechen. Die RAFAD-Garantie erlaubte es auch, die Vermittlerrolle zu spielen, indem sie das Nyesigiso-Netz mit der Lokalbank in Verbindung brachte.

\section{Eine Lokalbank, die in der Entwicklung ihres Landes ihren Vorteil findet}

Die lokale Finanzinstitution, la Banque Internationale pour le Mali (BIM), hat sich bei diesem Geschäft stark engagiert. Sie hat in der Tat nach Verhandlungen zwischen dem Nyesigiso-Netz, RAFAD und der BIM von Anfang an 50\% der Risiken gedeckt. Somit liess sich dank der RAFAD-Garantie eine Hebelwirkung von 2 erzielen, die in der Folge sogar auf 3,3 anstieg.

Dieses Garantiebeispiel hat gezeigt, dass zwei Bankensysteme, die verschiedene Logiken und Interessen verfolgen (Nyesigiso, das seine Kreditvergabekapazität erhöhen möchte, und die BIM, die ihre Überliquiditäten verwenden will) bestens zusammenarbeiten und zur sozio-ökonomischen Entwicklung des Landes beitragen können.

Die Wirkung der Garantie hat voll funktioniert, die Bank und das NyesigisoNetz betrachten sich heute als Geschäftspartner. Die Garantie wurde anschliessend dazu eingesetzt, die eventuellen Verlustrisiken oder den auf jahreszeitlichen Schwankungen beruhenden Liquiditätsmangel zu decken.

Da die Finanzlage sich mit einer Bruttospanne, die alle Betriebskosten ohne 
Subventionen deckt, konsolidiert hat, rechtfertigte sich der Nutzen einer RAFAD-Garantie nicht mehr. Daher hat Nyesigiso beschlossen, die Garantie zu annullieren und im Herbst 1999, acht Monate vor ihrer Fälligkeit, zurückzugeben.

\section{Indikatorentabelle}

\section{RAFAD-Garantie}

Ausstellung:

Annullierung:

Betrag:

Lokalbank:

Verwendung der Garantie:

Hebelwirkung:

Risikodeckung:

\author{
05.05.1997 \\ 19.10.1999 \\ 150 '000 SFR \\ Banque Internationale pour le Mali (BIM) - Bamako \\ Ausfallrisiko des Spar- und Kreditkassennetzes \\ 3.3 \\ $\checkmark 70 \%$ durch die Mitglieder der Kassen und die BIM, \\ 口 $30 \%$ durch RAFAD, vom 1. Franken an
}

\section{Soziale Auswirkungen}

Anzahl betroffener Personen: a Das Nyesigiso-Netz umfasste 1999 rund 50'000 Mitglieder. 口 Ausserdem hat Nyesigiso 1997 5'828 Kredite gewährt, die zumgrössten Teil an Mikro-Familienbetriebe vergeben werden.

- Man kann annehmen, dass Nyesigiso wahrscheinlich eine grosse Anzahl Arbeitsplätze bei der lokalen Bevölkerung aufrechterhalten hat.

Anzahl betroffener Frauen: $\quad$ Ein sehr hoher Anteil der Kundschaft, bei den Krediten für Gruppen ein Mehrheitsanteil. Zudem wurden spezifische Programme zur Unterstützung mittelloser Landfrauen eingerichtet. Schliesslich wird das Netz von einer Frau geleitet.

Sozialpolitik des Partners: $\quad$ Die Mitglieder des Netzes nehmen in demokratischer Weise (mit einer Stimme pro Mitglied) an der Ausrichtung und Kontrolle der Kassen teil. Ausbildungseffekt: Das Netz bietet jeder lokalen Kasse technische und finanzielle Unterstützung an.

\section{- Beispiel: Das RAFAD/FIG-System zur Unterstützung} der Kaffeeproduzenten in Peru

In Peru unterhält die Stiftung RAFAD seit rund zehn Jahren eine Partnerschaft mit der Genossenschaft Cooperativa Agraria Cafetalera (CAC) La Florida. Sie ist die letzte Kaffeeproduzenten-Genossenschaft der Provinz Chanchamayo und exportiert über ein Drittel ihrer Produktion auf den Markt des westlichen alternativen Handels. Mit den Garantien von RAFAD und dem Fonds International de Garantie (FIG) finanziert die Genossenschaft den Vermarktungsfonds für den Ankauf von Kaffee von ihren Mitgliedern.

Ein leidenschaftlicher Wille, die Genossenschaft nach langen Jahren der Unsicherheit wiederaufzubauen: In der Provinz Chanchamayo, in der La Florida ansässig ist, hat die Bevölkerung stark unter den Massakern gelitten, die von der Terrororganisation «Leuchtender Pfad» Anfang der 90er Jahre verübt worden waren. Neben den Terrorismusproblemen hat die Präsenz von Drogenhändlern in den letzten Jahren das in der Region herrschende Klima der Unsicherheit noch verstärkt. 
Paradoxerweise hat die Genossenschaft in diesem besonderen Kontext grosser Unsicherheit einen zweiten Anlauf genommen. Sie wurde 1966 gegründet, war aktiv und erfolgreich, doch zu Beginn der 90er Jahre, auf dem Höhepunkt der terroristischen Unruhen, hatte La Florida nur noch etwa 40 Mitglieder. Zudem erhielt die Genossenschaft zu jener Zeit infolge der Liberalisierung der Kaffeevermarktung nicht mehr die Unterstützung, die der Staat den Genossenschaften traditionell zukommen liess.

Eine wieder in vollem Wachstum befindliche Tätigkeit: Trotz dieser Umstände hat es der Wille und die Arbeit einer Gruppe besonders tatkräftiger Mitglieder der Genossenschaft ermöglicht, wieder auf die Beine zu kommen. Sie hat jetzt rund 700 aktive Mitglieder, steigert ihre Produktion Jahr für Jahr, und obwohl sie finanziell noch nicht unabhängig ist, erzielt sie dennoch aus dem Kaffeeexport Gewinne. La Florida löst ihre Probleme auch dadurch, dass sie sich auf eine Produktion von hoher Qualität konzentriert, die sie anschliessend teilweise (zu 30\%) auf dem Markt des westlichen Fairen Handels absetzt. Dank dieser Absatzmöglichkeiten kann sie mit überdurchschnittlichen Kaufpreisen rechnen.

Wenn es La Florida gelungen ist, sich wieder aufzurichten, so muss man dabei auch die Bedeutung der Hilfe hervorheben, die sie von Vereinigungen des Nordens erhalten hat. Traditionelle Akteure des «Fair Trade»-Bereichs wie Rothfos (Deutschland), aber auch Terre des Hommes (Genf) und SOS-Faim (Belgien) haben die Genossenschaft finanziell unterstützt. Die sehr aktive Genfer Organisation La Florida-Pérou bietet ihr ebenfalls bedeutende Unterstützung bei ihren sozialen und Vermarktungstätigkeiten an.

Ein Projekt, durch das die Lebensbedingungen der Gemeinschaft verbessert werden können: La Florida hat eine Reihe sozialer Infrastruktureinrichtungen, vor allem in den Bereichen Ausbildung, landwirtschaftliche Beratung, Gesundheit und Erziehung geschaffen. Neben der bedeutenden Stellung, die sie auf dem lokalen Kaffeemarkt insbesondere betreffend die Festsetzung der Preise einnimmt, bringt La Florida auch eine globale Vision der Entwicklung ein. Dadurch trägt sie dazu bei, ein für die Stabilität und den Wohlstand der 10'000 Familien in der Region günstiges Klima zu schaffen.

Der FIG, ein den Bedürfnissen von La Florida angepasstes Finanzinstrument: Die Notwendigkeit, sich an Hilfe von aussen zu wenden, wird ab 1990 besonders dringlich. Ohne weitere Unterstützung des Staates, durch die Terroraktivitäten zerrüttet, musste La Florida unbedingt eine Finanzierung auftreiben, um einen Vermarktungsfonds für den Kaffeeankauf von ihren Mitgliedern zu sichern. Da der Genossenschaft der Zugang zum lokalen Kredit ohne Garantieerbringung verwehrt war, hat sie sich dann an RAFAD gewandt. Eine erste Garantie wurde für die Ernte 1990 ausgestellt und ist seither jedes Jahr erneuert worden.

Im Jahr 2000 ist La Florida Mitglied des Fonds International de Garantie (FIG) geworden und hat sich dieser Genossenschaft aus Mitgliedern des Südens und des Nordens angeschlossen, deren Ziel es ist, Garantien für Partner des Südens zu gewähren. 
Der Rückgang der Kaffeepreise hat zu einer Inanspruchnahme der Garantie geführt, ohne jedoch den Konkurs der Organisation nach sich zu ziehen: Das Sinken der Kaffeepreise wirft für die Rentabilität der Genossenschaft Schwierigkeiten auf. 1992 hatte der Verfall der Kaffeekurse eine zweimalige Inanspruchnahme der Garantien in Höhe von insgesamt 50'000 SFR zur Folge. RAFAD's Vertrauen in den Willen und die Fähigkeiten von La Florida, sich wieder aufzurichten, ist niemals geschwunden. La Florida hat gezeigt, dass sie imstande ist, ihre Verpflichtungen einzuhalten, indem sie regelmässig die Schulden zurückzahlt, die sich aus der Inanspruchnahme der Garantien ergeben haben. Bis heute ist ihr Verschuldungsniveau bei ausländischen Geldgebern noch hoch, jedoch ist es der Genossenschaft gelungen, ihre grössten Schulden zeitlich umzuprogrammieren. Die Frage der internationalen Kaffeepreise, auf die die Genossenschaft keinen Einfluss hat, bleibt jedoch aktuell und stellt weiterhin eine grosse Sorge für diese Organisation dar.

\section{Indikatorentabelle}

\section{RAFAD-Garantie}

$\begin{array}{ll}\text { Ausstellung: } & 15.05 .2000^{17} \\ \text { Fälligkeit: } & 31.12 .2000 \\ \text { Betrag: } & 1255^{\prime} 000 \text { USD } \\ \text { Lokalbank: } & \text { Banco Continental - Lima } \\ \text { Verwendung der Garantie: } & \text { Lieferung und Vermarktung von Kaffee } \\ \text { Hebelwirkung: } & 1.4 \\ \text { Risikodeckung: } & \sqcup 72 \% \text { durch RAFAD (Rückbürgschaft der DEZA) und durch den FIG } \\ & \text { (davon 4\% FIG-Anteile von La Florida) } \\ & \sqcup 28 \% \text { durch Banco Continental, vom 1. Franken an }\end{array}$

\section{Soziale Auswirkungen}

Anzahl betroffener Personen: Die Genossenschaft umfasst rund 700 Kaffeeproduzenten. Sie schafft über 2'500 direkte Arbeitsplätze und hält durch die erlangten Finanzierungen indirekt 450 Arbeitsplätze aufrecht.

Anzahl betroffener Frauen: La Florida hat zwei «Frauenkomitees» mit rund 80 Mitgliedern eingesetzt, die ihre Aktivitäten mittels Kaffeebaumschulen finanzieren.

Sozialpolitik des Partners: Die Genossenschaft lässt ihren Mitgliedern Gesundheits-, Erziehungsund Rechtsberatungsdienste zukommen. Sie arbeitet auch mit Mandat, um die notwendigen Schritte für den Bau von Strassen, Brücken und Stromnetzen und für Bildungsdienste zu unternehmen.

Ausbildungseffekt: $\quad$ Eine Ausbildungsschule gibt Kurse für Produktion und Bodenbewirtschaftung mit hohen Qualitätskriterien. Ferner begleitet eine technische Unterstützung durch spezialisiertes Personal den für die Produzenten bestimmten Rotationsfonds. 


\section{WELCHE BILANZ LÄSST SICH AUS DER RAFAD/FIG-ERFAHRUNG ZIEHEN?}

Aus der Erfahrung der Stiftung RAFAD und des FIG kann eine Zwischenbilanz in Bezug auf den Einsatz der Garantie gezogen werden. Hierdurch lässt sich das Instrument der Bankgarantie mit seinen Erfolgen, Fehlschlägen, Möglichkeiten und Hindernissen besser umreissen.

Als hauptsächlicher Erfolg ist die Mobilisierung und die Erhöhung der Finanzmittel für den informellen Wirtschaftssektor zu unterstreichen. Die Verluste sind relativ gering und die Hebelwirkung ist akzeptabel. Ferner ist hervorzuheben, dass die Akteure der informellen Wirtschaft mittels der Garantie an den modernen Bankensektor herangeführt werden können.

Die grössten Schwierigkeiten beim Einsatz der Garantie liegen bei den Rahmenbedingungen (flaue Wirtschaftslage, hohe Zinssätze, Misstrauen der klassischen Finanzinstitutionen, Bestehen von Darlehen zu subventionierten Zinssätzen, usw.).

Ein weiteres grosses Problem sind die Betriebskosten eines internationalen Garantiefonds zur Deckung der Ausgaben, die mit der Prüfung der Dossiers, der Betreuung vor Ort, der Ausbildung, der Öffentlichkeitsarbeit, usw. verbunden sind. Wenn man die Eigenfinanzierung des Fonds erreichen und eine erträgliche und angemessene Kommission (zwischen 2.5 und 5\% des Garantiebetrags) für den Garantienutzer in Rechnung stellen will, so muss der Fonds einen relativ hohen Umfang (zwischen 4-6 Millionen SFR) erreichen, wovon 60-70\% für die Ausstellung von Garantien eingesetzt werden müssen. Dieser Betrag wird zur Zeit von RAFAD/FIG noch nicht erreicht, was das System noch vom Hilfesystem abhängig macht.

Die Möglichkeiten liegen vor allem in der Entwicklung des FIG. Sein Vorteil im Vergleich zum ursprünglichen System ist, dass der Garantienehmer, um eine Garantie zu erhalten, Mitglied der Genossenschaft FIG sein muss und somit Geschäftsanteile erworben haben muss. Dies sollte die Verantwortung und die Partizipation der Mitglieder beträchtlich erhöhen und folglich dazu beitragen, dass der FIG zu einer Institution wird, bei der die Partner des Südens aktiv an den Beschlüssen teilnehmen. Durch die Rechtsform der Genossenschaft, die nach dem System «ein Mitglied - eine Stimme» funktioniert, sollte es möglich sein, den FIG auf demokratische Art und Weise zu verwalten, unabhängig vom Betrag der Geschäftsanteile, die von den Mitgliedern gehalten werden.

Ein weiterer Vorteil des FIG ist die bessere Verteilung des Risikos innerhalb der Institution, das nach und nach durch die Geschäftsanteile des Garantiebegünstigten (der auch Genossenschaftsmitglied ist) gedeckt wird, ferner durch die (aus Schenkungen stammenden) Reservemittel und schliesslich durch das Geschäftskapital, das die gesamten Mitgliederanteile engagiert.

In einer weiter gefassten Perspektive kann der FIG auch ein interessantes Instrument für Entwicklungsorganisationen im Norden sein, welche

口 Mitglied des FIG werden können und hierdurch «nützliche und solidarische» Anlagen tätigen können;

- Anteile erwerben können, um ihre Partner des Südens teilhaben zu lassen, indem sie ihnen einen «Garantie-Schalter» eröffnen;

- sich am Erwerb von Anteilen für Partner beteiligen können, die Mitglied des FIG werden möchten, aber nicht über genügend Eigenmittel verfügen (NordSüd-Patenschaft). 
Der FIG bietet auch Sparern, die niemals eine Garantie beantragen werden, die Möglichkeit, (nicht vergütete, aber wieder veräusserbare) Anteile zu erwerben und dadurch die Finanzkapazität des FIG zu stärken.

Als wesentlichstes Hindernis für die Entwicklung des Systems ist die Verschlechterung der sozio-ökonomischen Rahmenbedingungen zu nennen, insbesondere die nationalen und internationalen Finanzkrisen, die auftreten können. Ein weiterer Hemmschuh für die Entwicklung des Systems kann die Politik der internationalen Entwicklungshilfeagenturen sein, die im informellen Wirtschaftssektor mit viel Fingerspitzengefühl intervenieren sollten, indem sie die Zuschüsse und subventionierten Kredite nur für ganz besondere soziale Situationen einsetzen.

Auf lokaler Ebene muss ein günstiger (gesetzgeberischer, institutioneller) Rahmen gegeben sein, um es den lokalen Akteuren zu erlauben, in Erscheinung zu treten und ihre Aktivitäten mit der grösstmöglichen Sicherheit entwickeln zu können. Die Hauptherausforderung für den FIG besteht dann darin, wirtschaftliche Projekte auf der Ebene der informellen Wirtschaft zu identifizieren, die dank Garantie und Kredit einen hohen Wertzuwachs in wirtschaftlicher, finanzieller und sozialer Hinsicht hervorbringen, dies mit dem Ziel, die Armut im Lande zu reduzieren.

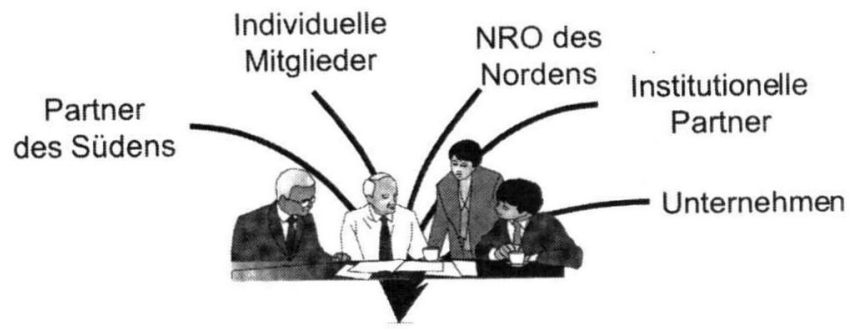

Internationaler Garantiefonds

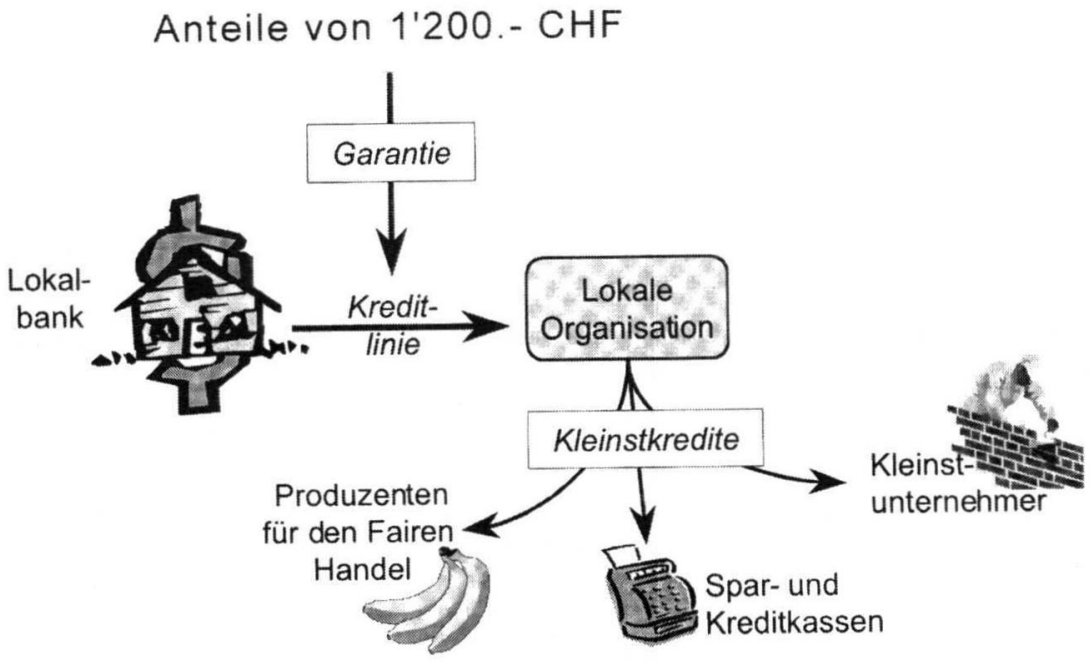




\section{EINIGE ABSCHLIESSENDE BETRACHTUNGEN: IST DIE BANKGARANTIE EIN INSTRUMENT ZUR FÖRDERUNG DER MIKROFINANZ?}

Diese Frage ist Gegenstand einer umfassenden Debatte mit mehreren in den letzten Jahren veröffentlichten Studien ${ }^{18}$, die grundsätzlich zwei Tendenzen aufzeigen. Auf der einen Seite sind die Gegner der Bankgarantie der Ansicht, dass diese keine Vorteile bringt, dass die Garantiesysteme langfristig finanziell nicht unabhängig sind und dass es besser wäre, die Bankvorschriften so zu ändern, dass die Transaktionskosten und der Kredit an die Kleinst- und Kleinunternehmen gesenkt werden. Auf der anderen Seite sind die Anhänger der Garantiesysteme der Meinung, dass diese unter bestimmten Bedingungen ein Instrument zur Förderung der Mikrofinanz darstellen können.

Parallel zu dieser Debatte beginnen Geschäftsbanken in den Entwicklungsländern überraschenderweise, sich für den Mikrofinanzsektor zu interessieren, während sie bislang angesichts des «Risikofaktors», der hohen Transaktionskosten und des Mangels an angemessenen Sicherheitsleistungen gezögert hatten. Banken in Asien (wovon einige den NRO sehr nahe stehen) haben in diesem Sektor in den letzten Jahren bedeutende Gewinne erzielt. Der verstärkte Wettbewerb auf ihrem traditionellen Markt hat sicher etwas mit dieser Annäherung zu tun, umso mehr als gewisse Mikrofinanzinstitutionen aus Finanzmethodologien Nutzen gezogen haben, die es ihnen erlauben, den Kreditleistungen der traditionellen Banken Konkurrenz zu machen, wobei sich einige sogar in reglementierte «Mikrobanken» verwandeln und ebenfalls Mikrokrediterleichterungen anbieten. Wir stellen somit eine sich abzeichnende Bewegung des Zusammengehens der traditionellen Banken und der Mikrofinanzinstitutionen auf dem potenziell einträglichen Mikrofinanzmarkt fest, dessen Nachfrage gegenwärtig bei weitem nicht durch die bestehenden Finanzinstitutionen gedeckt wird.

Innovative Instrumente, wie die Garantiesysteme, können zur stabilen Finanzierung der leistungs- und lebensfähigen Mikrofinanzinstitutionen beitragen, die eine angemessene Sicherheitsleistung bei den Leihbanken benötigen, wobei die Instrumente es diesen Banken erlauben, sich mit dem Mikrofinanzsektor vertraut zu machen, und es den Mikrofinanzinstitutionen ermöglichen, die Erfordernisse und Leistungskriterien der Geschäftsbanken zu erlernen und letztendlich eine gewisse Glaubwürdigkeit auf dem Kapitalmarkt zu erlangen.

In diesem Kontext hat uns die Erfahrung bei RAFAD gezeigt, dass der Erfolg eines Garantiesystems von der genauen Einhaltung einer Reihe von Bedingungen abhängt ${ }^{19}$ :

1. Klarheit bei der Definition des Kreditempfängers.

2. Ein Finanzsektor, der nicht an einer ungenügenden Liquidität leidet.

3. Ein Garantiesystem, das glaubwürdig sein muss, um wirksam zu sein.

4. Ein Garantiesystem, das dank des Vertrauens der Geschäftsbank eine finanzielle Hebelwirkung erreicht, was mit der Zeit eine Entwicklung des Kredits ermöglicht, der die Inanspruchnahme dieses Garantiesystems überflüssig macht.

18. Siehe Anhang 1 : Bibliographie zu den Garantiesystemen.

19. Las garantías bancarias, un instrumento financiero en debate, Dominique Lesaffre/RAFAD, FOLADE, 1.-3. Dezember 1997, Lima, Peru, S. 2-3. 
5. Ein effizientes Garantiesystem, das die finanzielle Unabhängigkeit anstrebt, seine mittelfristigen Betriebskosten und seine langfristigen Verluste deckt.

6. Ein Garantiesystem, das um dauerhaft zu sein, attraktiv sein muss sowohl für die Empfänger, die das Garantievolumen bestimmen werden, als auch für die Geschäftsbanken, die in einem liberalisierten finanziellen Rahmen mit vom Markt diktierten Zinssätzen operieren, und die Investoren, die den Garantiefonds finanzieren werden.

Wenn man auch die Schwierigkeiten anerkennen muss, die im Verlauf der Evaluationen von Garantiesystemen aufgetreten $\operatorname{sind}^{20}$, so ist es dennoch nützlich, die wesentlichen Probleme, auf die Garantiefonds in der Vergangenheit gestossen sind, zusammenzufassen ${ }^{21}$ :

- Seitens der Geschäftsbanken konnte man zuweilen ein gewisses Trägheitsverhalten feststellen, das sich durch eine oberflächliche Prüfung des Kreditdossiers oder des «subjektiven" Risikos ausdrückte, wobei die riskantesten Kreditangebote den Garantiesystemen überlassen wurden.

- Seitens der Darlehensnehmer: das Absinken der Rückzahlungsraten aufgrund des Wissens, dass der Kredit abgesichert ist (durch Disziplinmangel beeinträchtigte Kreditkultur, besonders wenn das System von der westlichen Entwicklungszusammenarbeit finanziert wird).

- Die Schwierigkeit, die Rolle der Garantie genau zu definieren und zu messen: spricht man vom Volumen neuer Darlehen, die aufgrund der Garantie gewährt wurden, oder von Krediten mit höheren Beträgen, oder auch von niedrigeren Zinssätzen, die dank der Garantie erlangt wurden?

- Die im allgemeinen negative Erfahrung (Ineffizienz, schlechte Ergebnisse), was die öffentlichen Garantiefonds betrifft, die oft in zentralistischer und bürokratischer Weise und mit festen Zielen verwaltet werden, die Bestandsbeschränkungen unterworfen sind.

- Die negative Erfahrung gewisser Garantiesysteme für subventionierte Kredite oder Haushaltsausgaben der Regierung, mit dem Risiko der Dekapitalisierung in Fällen nicht zugewiesener Haushaltsmittel oder hoher Nichtrückzahlungsraten.

- Die Schwierigkeit, den Garantiesystemen, die noch häufig von Subventionen abhängig sind, einen dauerhaften Charakter zu verleihen. In diesem Kontext muss die Kontinuität der Garantiesysteme auf lange Sicht analysiert werden.

Anhand der Aufzählung der oben aufgeführten Schwierigkeiten wird klar, dass es sich um Garantiesysteme handelt, die oft mit dem Einsatz subventionierter Kredite verbunden sind, welche in den meisten Fällen von den Banken selbst oder den staatlichen Stellen verwaltet werden. Bei diesen Systemen werden die lokalen Organisationen, welche die Besonderheiten des informellen Wirtschaftssektors kennen, und die wirtschaftlichen Akteure bei der Festlegung der Politik und der Anwendung der Garantiesysteme nicht mit einbezogen.

Parallel zu diesem System kann man das Entstehen einer zweiten Generation von Garantiesystemen feststellen, zu denen das RAFAD-System gehört. Diese

20. Diese Schwierigkeiten sind bedingt durch: a) einen Mangel an systematischen quantitativen Daten, b) einen zu kurzen Erfahrungshorizont in Bezug auf die Aktivitäten, c) ein Fehlen fundierter Studien in Anbetracht der konzeptuellen und methodologischen Probleme und der sich ergebenden hohen Kosten.

21. Las garantías bancarias, un instrumento financiero en debate, Dominique Lesaffre/RAFAD, FOLADE, 1.-3. Dezember 1997, Lima, Peru, S. 4. 
Systeme geben Krediten den Vorzug, die den Marktzinssätzen folgen, arbeiten eng mit den Organisationen zusammen, die mit der informellen Wirtschaft verbunden sind, legen Nachdruck auf Disziplin bei der Kreditrückzahlung und auf die Mobilisierung der Spargelder, die bei der Risikoverteilung als lokaler Garantiefonds dienen werden. Diese neuen Systeme legen den Schwerpunkt auch auf die Bedeutung der Partnerschaft mit der Lokalbank und ihr Interesse, den Mikrofinanzsektor zu erforschen, um eine Hebelwirkung zu ermöglichen. Fortan ergeben sich aus den Lehren, die aus den Erfahrungen mit den Bankgarantien in der Vergangenheit gezogen wurden, gewisse Regeln, die einzuhalten sind, um den Erfolg dieser neuen Generation von Garantiesystemen zu gewährleisten ${ }^{22}$.

Unter den wichtigsten Prinzipien sind folgende zu nennen:

- Die Garantie muss beschränkt sein und als befristet betrachtet werden.

- Die Garantie muss klar definiert sein, gut begriffen werden und den spezifischen Bedürfnissen der Begünstigten angepasst sein, als Ergebnis eines reiflich überlegten gegenseitigen Engagements, das zwischen Garanten, Banken und Mikrofinanzinstitutionen ausgehandelt wird. Insbesondere sollen die Darlehensgeber die Garantie erst von dem Zeitpunkt an als «attraktiv » ansehen, wenn sie überzeugt sind, dass das dem Kredit zugrundeliegende Projekt wirtschaftlich tragfähig ist, und - von sich aus - alles Nötige tun werden, um sich der Kreditrückzahlung zu vergewissern.

- Die Garantien erfordern eine regelmässige Betreuung, die es erlaubt, die Leistung der Mikrofinanzinstitutionen, vor allem betreffend die Darlehensrückzahlungsraten, den Cash Flow und die Auszahlung der Mikrokredite ständig zu messen.

- Die Garantien des «intermediären» Typs (wie Beispiel Nr. 1 «yesigiso») sind den «individuellen» Garantien oder den «Bestandsgarantien» (für die Gesamtheit einer spezifischen Gruppe) vorzuziehen ${ }^{23}$

- Der Erfolg der Garantie hängt von der internen Managementkapazität der Mikrofinanzinstitutionen ab. Eine institutionelle Stärkung ist unerlässlich, die darauf abzielt, ihr Finanzverwaltungssystem, die Qualität ihres Bestandes und das Risikomanagement zu verbessern.

- Die Dokumentation und die Verbreitung der «guten Verfahrensweisen» in diesem Bereich sind wesentlich für den Lernprozess der Banken wie auch der Mikrofinanzinstitutionen.

- Der Erfolg sollte nicht nur in Form von zusätzlichen mobilisierten Finanzmitteln gemessen werden, sondern auch anhand von indirekten Vorteilen, wie beispielsweise das erhöhte Vertrauen der Geschäftsbanken, eine bessere Finanzdisziplin der Mikrofinanzinstitutionen, finanzielle Transparenz, usw.

- Die Annäherung zwischen dem Mikrofinanzsektor und dem Bankensektor wird nicht nur von der Garantie abhängen, sondern auch vom echten Willen und vom Interesse zur Zusammenarbeit bei den Banken sowie von der Disziplin und der finanziellen Transparenz der Mikrofinanzinstitutionen.

22. Contributions au séminaire sur le thème «Systèmes de garantie dans un contexte de financement au développement", organisé par ADA les 7 et 8 Septembre 1998, Mia Adams, ADA, Dialogue, numéro 15, November 1998, S. 7-10.

23. Vgl. auch in der Bibliographie die Beispiele von gewährten Garantien von: ACCION International, Women's World Banking, Appui au Développement Autonome (ADA Luxemburg), SOS Faim. 


\section{BIBLIOGRAPHIE ZU DEN GARANTIESYSTEMEN}

ACCION INTERNACIONAL. A Decade of Guaranteeing Succes. Accion Internacional Bulletin, vol. 30, no.1, Winter 1995.

ADA. Micro-entreprises, Actes du séminaire organisé à Munsbach les 25-26-27 juin. Luxembourg, 1996.

ADA. Microfinance, Actes du séminaire organisé le $1^{\text {er }}$ juillet à Luxembourg. Luxembourg, 1997.

Balkenhol et C. Lecointre. Les banques et la PME en Afrique de l'Ouest. Problèmes de financement: seize études de cas. Paris, L'Harmattan, 1996.

Bastiaenen, Michiel and Peter van Rooij. Guarantee Funds and NGOs: Promise and Pitfalls. A Review of the key Issues. Working paper no. 18. Geneva, ILO, 43 p.

Baydas, Mayada., and others. Commercial Banks in Micro-finance: New Actors in the Micro-finance World. Microenterprise Best Practices 1997.

Brugger, Ernst A., and others. Impact of the FUNDES guarantee programs on small businesses in Latin America, 1996.

Castellanos, Jorge. «The Financial Supervision of Loan Guarantors». The Financier, vol.4 Issue $1 \& 2$ Feb-May 1997, pp. 34-43.

CGAP. Microcredit Interest Rates. «Occasional Papers», 1996.

Craig, Churchill. Regulation and Supervision of Micro-finance Institutions: Case Studies, The microfinance Network Occasional Paper no. 2. 1997.

Credit Guarantees Bibliography. Devfinance, Ohio University, 1998.

Demdoum, Samira. Les garanties bancaires: outils de développement et de contrôle des ONG. Mémoire de fin d'études. Université de Mons-Hainaut, 1997, 80 p.

Diouf, M. La coopération: une nécessité entre les banques et les opérateurs de la micro-finance. ADA Dialogue no. 8 pp .29-25.

Ditchter, T.W. «Questioning the Future of NGO'S in Micro-finance». ADA Dialogue no. 11, pp. 23-28.

Gallardo, Joselito and others. A commercial bank's micro-finance programs the case of Hatton National Bank in Sri Lanka. World Bank discussion paper, 369 p.

Gilbert, Pierre Luigi. «Pricing Credit Risk in Loans and Guarantees». The Financier, vol. 3 Issue 4/5 Nov-Dec. 1996, pp. 26-32.

Graham Bannock and Partners, Ltd. Credit Guarantee Schemes for Small Business Lending: A Global Perspective, Volume 1 - Main Report, April 1997.

Gudger, Michael. «The sustainability of credit guarantee systems», Small Enterprise Development, Vol. 8, No. 2, June 1997.

Gudger, Michael. Credit Guarantees: An Assessment of the State of Knowledge and new Avenues of Research, FAO, (November 1997, Draft).

Hatakeyama, Michiko, S. and others. «Credit Guarantee systems for Small and Medium Enterprise in Some Asian Countries». The Financier, vol.4 Issue 1\&2, Feb-May 1997, pp. 67-23.

Kaboré, Noël. «Relations between Conventional Agricultural Banking and Decentralized Financial Systems: Two Approaches. Current situation and outlook in Burkina Faso ». Techniques Financières \& Développement, Special Supplement, 1997, pp. 21-26

Le risque dans l'attribution de garanties aux organisations de développement du secteur non-formel du Tiers Monde. Edité par Fernand Vincent, RAFAD, Gèneve, 1995, 59 p.

Levistsky, Jacob. Credit Guarantee Funds and Mutual Guarantee Systems? Small Enterprise Development, vol. 4 no .2, June 1993.

Levitsky, Jacob and Prasad Ranga N. «Credit Guarantee Schemes for Small and Medium Enterprises», The Financier, vol. 4 Issue 1\&2, Feb-May 1997, pp. 62-73.

Llorens, Juan Luis. «Loan Guarantee System for SMEs in Europe». The Financier, Vol. 4 Issue 1\&2, Feb-May 1997, pp. 74-85.

Malhotra, Mohini. «Micro-finace: The New Emerging Market? CGAP, 1997.

Marulande de García, Beatriz. National Guarantee Fund of Colombia. The Financier, vol. 4 Issue 1\&2 Feb-May 1997, pp. 44-50.

McGuire, Paul B. and Jhon D. Conroy. «Bank-NGO Linkages and the transaction costs to the lending to the poor through groups ", Small Enterprise Development, vol.8 no.1, pp. 4-15.

Meyer, Richard L. and Nagarajan Geetha. Credit Guarantee Schemes for Developing Countries: Theory, Design and Evaluation Center for Economic Growth, Ohio University, 1996.

Mosley, Paul. Metamorphosis from NGO to Commercial Bank: the case of Bancosol in Bolivia. Institute for Developpement Policy and Management, Working Paper, no. 4, 1993, 45 p.

Oehring, Eckart. «The FUNDES Experience with Guarantee Systems in Latin America: Model, Results and Prospects ». The Financier, vol.4 Issue 1\&2 Feb-may 1997, pp. 57-61. 
Otero, Maria. Accessing Commercial Capital for Micro-finance: The ACCION Experience. Speech delivered at a seminar on Rural Finance at the World Bank, October 241996.

Pomareda, C. and Max Soto. Regulación Organizaciones Financieras No Convencionales y Microfinanciamiento: estado de la discusión. Folade, Costa Rica, 1977.

Round Table on Credit Guarantee Systems: International Experiences and Lessons for Latin American and the Caribbean. Inter-American Development Bank, Washington D.C., June 27-28, 1996.

Rowlatt, Amanda. «Micro-finance - Involvement Banks», Small Enterprise Development, vol. 8, no. 2, 1997.

Shari Berenbach and Craig Churchill. Risk Profile and Regulatory Approaches to Micro-finance Institutions, in «Regulation and Supervision Micro-finance Institutions. Experience from Latin America, Asia and Africa». The Micro-finance Network Occasional Paper no.1, pp. 19-30.

Smith, Anne-Marie. Bank Dagang Bali : A Profitable Commercial Bank in Micro-finance. CGAP, 1977.

Stearns, Katherine. Leverage or Loss Guarantee Funds and Microenterprise, ACCION International, Monograph Series No. 8.

Thembani International Guarantee Fund (TIGF): Banking on South Africa's Democratic Development. Johannesburg, TIGF Office, 1996.

Vogel, Robert C. and Dale W. Adams. Cost and Benefits of Loan Guarantee Programs. The Financier, vol.4 Issue 1\&2 Feb-May 1997, pp. 22-29.

Vogel, Robert C. and Dale W. Adams. Rationale for Establishing Credit Guarantee Systems, Paper Presented at the Round Table on Credit Guarantee Systems: International Experiences and Lessons for Latin America and the Caribbean, Inter-American Development Bank, Washington D.C. June 2728,1996, $15 \mathrm{p}$.

Women's World Banking. The Missing Links: Financial Systems that Work for the Majority. WWB, 1995, 38p.

Young, Robin; Lara Goldmark, and others. Micro-finance Guarantees: A Basic Primer and Review of Experiences in Latin America and the Caribbean, Inter-American Development Bank, Microenterprise Unit, December, 1997, 29 p. 